\title{
LoCuSS: The infall of X-ray groups on to massive clusters
}

\author{
C. P. Haines, ${ }^{1,2 \star}$ A. Finoguenov, ${ }^{3,4 \star}$ G. P. Smith, ${ }^{5 \star}$ A. Babul, ${ }^{6}$ E. Egami, ${ }^{7}$ \\ P. Mazzotta, ${ }^{8}$ N. Okabe, ${ }^{9}$ M. J. Pereira,${ }^{7}$ M. Bianconi, ${ }^{5}$ S. L. McGee, ${ }^{5}$ F. Ziparo, ${ }^{5}$ \\ L. E. Campusano ${ }^{2}$ and C. Loyola ${ }^{2}$ \\ ${ }^{1}$ INAF - Osservatorio Astronomico di Brera, via Brera 28, I-20121 Milano, Italy \\ ${ }^{2}$ Departamento de Astronomía, Universidad de Chile, Casilla 36-D, Correo Central, Santiago, Chile \\ ${ }^{3}$ Max-Planck-Institut für extraterrestrische Physik, Giessenbachstraße, D-85748 Garching, Germany \\ ${ }^{4}$ Department of Physics, University of Helsinki, Gustaf Hällströmin katu 2a, FI-0014 Helsinki, Finland \\ ${ }^{5}$ School of Physics and Astronomy, University of Birmingham, Edgbaston, Birmingham B15 2TT, UK \\ ${ }^{6}$ Department of Physics and Astronomy, University of Victoria, 3800 Finnerty Road, Victoria, BC V8P 1A1, Canada \\ ${ }^{7}$ Steward Observatory, University of Arizona, 933 North Cherry Avenue, Tucson, AZ 85721, USA \\ ${ }^{8}$ Dipartimento di Fisica, Università degli Studi di Roma 'Tor Vergata', via della Ricerca Scientifica 1, I-00133 Roma, Italy \\ ${ }^{9}$ Department of Physical Science, Hiroshima University, 1-3-1 Kagamiyama, Higashi-Hiroshima, Hiroshima 739-8526, Japan
}

Accepted 2018 March 4. Received 2018 February 25; in original form 2017 September 8

\begin{abstract}
Galaxy clusters are expected to form hierarchically in a $\Lambda$ cold dark matter $(\Lambda \mathrm{CDM})$ universe, growing primarily through mergers with lower mass clusters and the continual accretion of group-mass haloes. Galaxy clusters assemble late, doubling their masses since $z \sim 0.5$, and so the outer regions of clusters should be replete with accreting group-mass systems. We present an XMM-Newton survey to search for X-ray groups in the infall regions of 23 massive galaxy clusters $\left(\left\langle M_{200}\right\rangle \sim 10^{15} \mathrm{M}_{\odot}\right)$ at $z \sim 0.2$, identifying $39 \mathrm{X}$-ray groups that have been spectroscopically confirmed to lie at the cluster redshift. These groups have mass estimates in the range $2 \times 10^{13}-7 \times 10^{14} \mathrm{M}_{\odot}$, and group-to-cluster mass ratios as low as 0.02 . The comoving number density of X-ray groups in the infall regions is $\sim 25 \times$ higher than that seen for isolated X-ray groups from the XXL survey. The average mass per cluster contained within these $\mathrm{X}$-ray groups is $2.2 \times 10^{14} \mathrm{M}_{\odot}$, or $19 \pm 5$ per cent of the mass within the primary cluster itself. We estimate that $\sim 10^{15} \mathrm{M}_{\odot}$ clusters increase their masses by $16 \pm 4$ per cent between $z=0.223$ and the present day due to the accretion of groups with $M_{200} \geq 10^{13.2} \mathrm{M}_{\odot}$. This represents about half of the expected mass growth rate of clusters at these late epochs. The other half is likely to come from smooth accretion of matter not bound within haloes. The mass function of the infalling X-ray groups appears significantly top heavy with respect to that of 'field' X-ray systems, consistent with expectations from numerical simulations, and the basic consequences of collapsed massive dark matter haloes being biased tracers of the underlying large-scale density distribution.
\end{abstract}

Key words: galaxies: clusters: general-galaxies: groups: general-dark matter-large-scale structure of Universe $-\mathrm{X}$-rays: galaxies: clusters.

\section{INTRODUCTION}

A key prediction of $\Lambda$ cold dark matter $(\Lambda \mathrm{CDM})$ cosmological models is that structure formation occurs hierarchically, whereby dark matter (DM) haloes grow via the continual accretion of lower mass systems. More massive haloes thus on average form later than less massive haloes. Galaxy clusters as the most massive collapsed

^E-mail: chris.haines@oa-brera.inaf.it (CPH); alexis.finoguenov@ helsinki.fi (AF); gps@star.sr.bham.ac.uk (GPS) haloes form latest, doubling their masses on average since $z \sim 0.5$ (Boylan-Kolchin et al. 2009; Gao et al. 2012), and are also the most dynamically immature. This manifests itself in 40 per cent of local clusters showing clear substructure in their X-ray emission (Jones \& Forman 1999; Chon, Böhringer \& Smith 2012; Mann \& Ebeling 2012), and a higher fraction with substructure in their underlying mass distributions inferred from gravitational lensing (Smith et al. 2005; Martinet et al. 2016).

The abundances and rates of growth of galaxy clusters thus represent sensitive probes of cosmology (Voit 2005; Henry et al. 2009; Kravtsov \& Borgani 2012), providing constraints on the primary cosmological parameters $\left(\Omega_{\mathrm{m}}, \Omega_{\Lambda}, \sigma_{8}, w_{0}\right)$ that are competitive 
with, and complementary to, those from supernovae, CMB, and baryon acoustic oscillations (Allen, Evrard \& Mantz 2011). While measurements of the local abundance and mass function (MF) of clusters have been used to jointly constrain the cosmic matter density $\Omega_{\mathrm{m}}$ and the amplitude of density perturbations $\sigma_{8}$ (e.g. Schuecker et al. 2003; Planck Collaboration XX 2014a), the rate of growth of massive clusters provides a window on the form and content of dark energy via the impact of cosmic acceleration on both structure formation and the distance-redshift relation (Mantz et al. 2010). Vikhlinin et al. (2009) compared the cluster mass function at $z \sim 0.5$ with that of present day clusters, finding an average mass growth of 75-80 per cent for massive clusters between $z \sim 0.5$ and 0.05 . This evolution maps the growth of structure and provides strong constraints on the dark energy density of the Universe with $\Omega_{\Lambda}=0.83 \pm 0.15$ (i.e. non-zero at $>5 \sigma$ significance) and its equation-of-state parameter $w_{0}=-1.14 \pm 0.21$, assuming a constant $w$ and a flat universe.

The merger histories of DM haloes and the mass functions of the progenitor haloes that are accreted by the primary cluster halo have been derived analytically using the extended PressSchechter formalism (EPS; Bond et al. 1991; Bower 1991; Lacey \& Cole 1993) and through cosmological $N$-body simulations (Lemson \& Kauffmann 1999; Governato et al. 1999; Giocoli, Tormen \& van den Bosch 2008) with good agreement between the two approaches. Interestingly, the unevolved subhalo mass function $\mathrm{d} N\left(m_{\text {sub }} / M_{0}\right) / \mathrm{d} \ln \left(m_{\text {sub }} / M_{0}\right)$, which parametrizes the masses of the progenitor subhalos $\left(m_{\text {sub }}\right)$ at the time they were accreted on to the primary cluster halo, has been found to be universal, with no dependency on the primary halo mass $M_{0}$, redshift (van den Bosch, Tormen \& Giocoli 2005; Giocoli, Tormen \& van den Bosch 2008).

Collapsed DM haloes are biased tracers of the underlying matter distribution, with the most massive haloes forming from the highest peaks in the primoridal linear density field. The EPS provides robust predictions for this bias as an increasing function of peak height $v$ or equivalently halo mass (Mo \& White 1996; Tinker et al. 2010). This leads to a systematic variation of halo mass function with large-scale $(\sim 10 \mathrm{Mpc})$ environment, with high-mass haloes overrepresented in high-density regions (Mo \& White 1996), a prediction that is reproduced well in $N$-body simulations (Governato et al. 1999; Lemson \& Kauffmann 1999; Faltenbacher, Finoguenov \& Drory 2010). As clusters are preferentially located at the centres of largescale overdensities, the MF of haloes in their surroundings, and which will subsequently be accreted on to them, should be biased towards higher mass systems.

The late assembly of clusters implies that there must be numerous group-mass systems in their outskirts in the process of being accreted. This motivates an X-ray survey covering the infall regions of a representative sample of massive clusters aimed at detecting these infalling group-mass systems and estimating their masses, with the ultimate objectives of estimating how much mass they contribute to the growth of the cluster through accretion, and whether their MF is indeed significantly biased with respect to more typical regions of the Universe. A key advantage of detecting these groups from their extended X-ray emission is that they can be unambiguously identified as massive virialized DM haloes. In contrast, a purely optically selected group sample (Ragone et al. 2004; Lemze et al. 2013) could be biased by the inclusion of non-virialized systems or chance lineof-sight (LOS) projections (O'Sullivan et al. 2017; Pearson et al. 2017), the likelihood of which are dramatically increased in the vicinity of rich clusters.

Many massive clusters at $z \sim 0.2$ have been observed by XMMNewton with exposure times $\gtrsim 10 \mathrm{ksec}$, sufficient to obtain reliable temperature, gas density, and mass profiles out to $r_{500}$ and $M_{500}$ mass estimates accurate to 10-25 percent (Zhang et al. 2007; Martino et al. 2014). These depths also permit the detection of galaxy groups at $z \sim 0.2$ down to masses of $\sim 2-3 \times 10^{13} \mathrm{M}_{\odot}$. While the intracluster medium (ICM) of the primary cluster is usually only detectable out to $\sim r_{500}$, the 30 arcmin field of view of XMM-Newton provides coverage out to $\sim 1.5-2.0 r_{200}$ and enabling infalling groups in the cluster outskirts to be detected.

In this paper, we present a search for X-ray groups in existing $X M M-$ Newton $\mathrm{X}$-ray observations targeting 23 massive clusters at $z \sim 0.2$ from the Local Cluster Substructure Survey (LoCuSS), for which highly complete stellar mass limited optical spectroscopy is available from the Arizona Cluster Redshift Survey (ACReS; Haines et al. 2013, 2015, Pereira et al. in preparation).

With this $X M M$ group sample, we derive the MF of galaxy groups infalling into massive clusters down to $M_{200} \sim 2 \times 10^{13} \mathrm{M}_{\odot}$, and estimate whether the accretion of these groups on to the clusters is sufficient to explain the expected mass growth of the clusters between $z \sim 0.2$ and the present day, or if further sources such as smooth accretion of DM are required. We examine the group-cluster mass ratio distribution and compare it to the unevolved subhalo $\mathrm{MF}$, whose universality is a key prediction of $\Lambda \mathrm{CDM}$ cosmological models (e.g. Giocoli et al. 2008).

We use a $\Lambda$ CDM cosmology with $\Omega_{\mathrm{M}}=0.27, \Omega_{\Lambda}=0.73$, and $H_{0}=72 h_{72} \mathrm{~km} \mathrm{~s}^{-1} \mathrm{Mpc}^{-1}$.

\section{THE CLUSTER SAMPLE, XMM DATA AND CONSTRUCTION OF THE GROUP CATALOGUE}

\subsection{The primary cluster sample}

The primary cluster sample for this study consists of all $23 \mathrm{X}$-ray luminous clusters within LoCuSS for which there is both high-quality $X M M-N e w t o n$ X-ray data and extensive spectrosopic coverage of cluster galaxies out to $\sim 3 r_{200}$ from ACReS to identify the most luminous member galaxies within the group and securely confirm its redshift.

LoCuSS is a systematic multiwavelength survey of $\sim 100 \mathrm{X}$-rayselected $\left(L_{\mathrm{X}} \geq 2 \times 10^{44} \mathrm{erg} \mathrm{s}^{-1}\right)$ massive clusters at $0.15 \leq z<0.30$, drawn from the ROSAT All Sky Survey catalogues (RASS; Ebeling et al. 1998, 2000; Böhringer et al. 2004). The ACReS subsample consists of the first batch of 30 clusters having wide-field optical imaging out to the virial radii from Subaru/Suprime-Cam and Hubble Space Telescope (HST) imaging of the cluster cores, enabling detailed mass maps combining weak- (Subaru) and stronglensing (HST) data. All 30 systems have excellent ancillary widefield, multiwavelength data including near-infrared imaging with United Kingdom Infra-Red Telescope/WFCAM $\left(J, K ; 52^{\prime} \times 52^{\prime}\right)$, Spitzer/MIPS $24 \mu \mathrm{m}$, and Herschel/PACS+SPIRE far-infrared photometry over $25 \times 25 \operatorname{arcmin}^{2}$ fields (Haines et al. 2010; Smith et al. 2010). The $L_{X}$ distribution of the ACReS subsample is statistically indistinguishable from the parent volume-limited sample of ROSAT clusters (Okabe et al. 2010). Of the 30 clusters covered by ACReS, 23 have existing $X M M-N e w t o n$ X-ray data suitable for detecting group-mass systems at the redshift of the cluster. These 23 systems form our primary cluster sample and are listed in Table 1.

A total of 20 out of the 23 clusters form part of the volumelimited 'high- $L_{X}$ ' LoCuSS sub-sample of 50 systems with $L_{X}(0.1-$ $2.4 \mathrm{keV}) / E(z) \geq 4.2 \times 10^{44} \mathrm{erg} \mathrm{s}^{-1},-25^{\circ}<\delta<+65^{\circ}$ and $n_{\mathrm{H}} \leq 7 \times 10^{20} \mathrm{~cm}^{-2}$ of Okabe et al. $(2013,2016)$ and Martino et al. (2014). Martino et al. (2014) extracted gas density and 
Table 1. The primary cluster sample. Columns. (1,2): cluster name and redshift. Column (3): ROSAT 0.1$2.4 \mathrm{keV}$ X-ray luminosity, except A689 ${ }^{a}$ which comes from Giles et al. (2012). Column (4) $M_{200}$ masses from the Chandra-XMM analysis of Martino et al. (2014) for the high- $L_{X}$ cluster sample, extending the mass profiles out to $r_{200}$, except for A665 and A2218c which come from Haines et al. (2013). Column (5) Weak lensing $M_{200}$ estimates from Okabe et al. (2016), except for A665, A689 and A2218 ${ }^{d}$, whose mass estimates come from Pedersen \& Dahle (2007); Okabe et al. (2010); Mahdavi et al. (2013).

\begin{tabular}{lcccr}
\hline $\begin{array}{l}\text { Cluster } \\
\text { name }\end{array}$ & $z$ & $\begin{array}{c}L_{\mathrm{X}} \\
\left(10^{44} \mathrm{erg} \mathrm{s}^{-1}\right)\end{array}$ & $\begin{array}{c}M_{200, \mathrm{X}} \\
\left(10^{14} \mathrm{M}_{\odot}\right)\end{array}$ & $\begin{array}{c}M_{200, \mathrm{WL}} \\
\left(10^{14} \mathrm{M}_{\odot}\right)\end{array}$ \\
\hline Abell 68 & 0.251 & 9.473 & $17.09 \pm 6.45$ & $9.24 \pm 1.74$ \\
Abell 115N & 0.192 & 8.895 & $6.24 \pm 1.18^{b}$ & $9.78 \pm 3.22$ \\
Abell 209 & 0.209 & 6.289 & $8.71 \pm 1.74$ & $17.71 \pm 2.90$ \\
Abell 267 & 0.229 & 8.569 & $9.28 \pm 2.83$ & $8.29 \pm 1.56$ \\
Abell 291 & 0.196 & 4.883 & $4.16 \pm 0.73$ & $7.82 \pm 2.01$ \\
Abell 383 & 0.189 & 4.559 & $4.46 \pm 1.20$ & $7.26 \pm 1.65$ \\
Abell 611 & 0.286 & 8.855 & $10.66 \pm 1.67$ & $12.82 \pm 2.52$ \\
Abell 665 & 0.183 & 9.837 & $11.71 \pm 2.79^{c}$ & $12.11 \pm 6.83^{d}$ \\
Abell 689 & 0.278 & $1.812^{a}$ & $4.34 \pm 0.45$ & $1.82 \pm 0.90^{d}$ \\
Abell 697 & 0.282 & 10.57 & $21.28 \pm 4.34$ & $13.53 \pm 3.49$ \\
Abell 963 & 0.204 & 6.390 & $8.92 \pm 2.01$ & $9.90 \pm 1.79$ \\
Abell 1689 & 0.185 & 14.07 & $14.93 \pm 3.69$ & $15.25 \pm 2.17$ \\
Abell 1758N & 0.279 & 7.514 & $18.21 \pm 3.59$ & $8.17 \pm 1.98$ \\
Abell 1763 & 0.232 & 9.317 & $14.50 \pm 3.18$ & $23.50 \pm 4.25$ \\
Abell 1835 & 0.252 & 24.48 & $21.46 \pm 5.68$ & $14.01 \pm 2.43$ \\
Abell 1914 & 0.167 & 10.98 & $10.70 \pm 2.41$ & $12.12 \pm 2.44$ \\
Abell 2218 & 0.173 & 5.554 & $6.73 \pm 1.60^{c}$ & $7.49 \pm 1.99^{d}$ \\
Abell 2219 & 0.226 & 12.73 & $21.22 \pm 4.94$ & $14.01 \pm 2.71$ \\
Abell 2390 & 0.229 & 13.43 & $22.13 \pm 7.11$ & $14.72 \pm 2.49$ \\
RXJ 1720 & 0.160 & 9.573 & $9.12 \pm 3.81$ & $7.26 \pm 2.37$ \\
RXJ 2129 & 0.234 & 11.66 & $7.19 \pm 0.86$ & $6.51 \pm 2.03$ \\
ZwCl 2089 & 0.235 & 6.786 & $2.67 \pm 0.35$ & $3.40 \pm 1.39$ \\
ZwCl 7160 & 0.257 & 8.411 & $6.02 \pm 1.37$ & $6.14 \pm 2.84$ \\
\hline
\end{tabular}

de-projected temperature profiles for each 'high- $L_{X}$ ' cluster from the same $X M M$ images analysed here, and derived total gravitational mass profiles and $M_{500}$ masses assuming hydrostatic equilibrium. These mass profiles were extended out to $r_{200}$ producing the $M_{200, X}$ mass estimates used here (Column 4), and have mean fractional uncertainties $\left\langle d M_{200} / M_{200}\right\rangle=0.238(0.093 \mathrm{dex})$. These uncertainties are almost double of those reported by Martino et al. (2014) for the $M_{500}$ masses $(0.052 \mathrm{dex})$, due to the need to extrapolate the mass profiles beyond $r_{500}$, but the ratios between these two masses vary little between systems, $\left(M_{500} / M_{200}\right)=0.648 \pm 0.094$. These 20 clusters also have updated weak-lensing mass measurements from Okabe et al. (2016) (Column 5) that are fully consistent on average with our X-ray mass estimates (Smith et al. 2016), with geometric mean mass ratio $\left\langle M_{200, \mathrm{X}} / M_{200, \mathrm{WL}}\right\rangle=0.985 \pm 0.106$.

Of the remaining three clusters, two (A665 and A2218) were only excluded from the high- $L_{\mathrm{X}}$ sample due to their declination $\left(\delta \sim+66^{\circ}\right)$, and we take their X-ray mass estimates $\left(M_{200, \mathrm{X}}\right)$ from Haines et al. (2013). These are based on fitting the phenomenological cluster models of Ascasibar \& Diego (2008) to a series of annular spectra extracted from deep Chandra data for each cluster (Sanderson \& Ponman 2010). The ROSAT $L_{\mathrm{X}}$ estimate of the final cluster Abell 689 satisfied our high- $L_{\mathrm{X}}$ selection and it was included in the ACReS sample of 30 clusters. It was excluded from the 'high- $L_{X}$ ' sample of Martino et al. (2014), however, as the Chan$d r a$ analysis of Giles et al. (2012) showed that its X-ray emission is dominated by a central BL Lac, and after excluding this central point source, the $L_{X}$ from the extended cluster emission falls below the LoCuSS survey limit. ACReS does confirm A689 as a cluster (with 338 members; Haines et al. 2015), and we estimate its mass from its updated $L_{\mathrm{X}}$ using the same $M_{200}-L_{\mathrm{X}}$ relation (Leauthaud et al. 2010) as used later for our XMM-detected groups (Section 2.4; equation 1).

A115 is a complex cluster merger with two approximately equal mass components A115N and A115S (Gutierrez \& Krawczynski 2005; Okabe et al. 2010). A115N is more X-ray luminous than A115S (Forman et al. 1981), and so we take A115N as our primary cluster, while Martino et al. (2014) only measured the mass of A115S. For consistency, we derive the $M_{200, \mathrm{X}}$ values for both A $115 \mathrm{~N}$ and A115S from the $L_{\mathrm{X}}$ estimates obtained in our analysis of the $X M M$ data (Section 2.2), using equation (1) (Section 2.4).

\subsection{Detecting extended sources in the $X M M$ data}

The details of the XMM-Newton observations and initial data reduction are summarized in Martino et al. (2014). After removal of energies affected by instrumental lines (as in Finoguenov et al. 2007), the $0.5-2 \mathrm{keV}$ band images from the pn and MOS detectors are in-field background subtracted and co-added. To detect and identify extended emission from X-ray groups, the $0.5-2 \mathrm{keV}$ band image for each cluster is then decomposed into unresolved and extended sources, using the wavelet scale-wise decomposition and reconstruction technique of Vikhlinin et al. (1998), employing angular scales from $8-64$ arcsec (2-16 pixels). The discrete pixellated data $c_{0}(i, k)$ from the $X M M$ image is progressively smoothed by convolution with a $5 \times 5$ matrix kernel $h(m, n)$, on scales that double from one step to the next, such that: $c_{j}(i, k)=$ $\sum_{m, n} h(m, n) c_{j-1}\left(i+2^{j-1} m, k+2^{j-1} n\right)$, where the indices $m$ and $n$ run from -2 to +2 . As the distance between sampled pixels doubles with each step, this is known as an à trous ('with holes') type of wavelet transform. The kernel used 
is based on the $B_{3}$ cubic spline scaling function, corresponding to the binomial coefficients $h(n)=(1 / 16,1 / 4,3 / 16,1 / 4$, $1 / 16)$ in one dimension. The wavelet transform at a smoothing level $j$ is then defined as $w_{j}(i, k)=c_{j-1}(i, k)-c_{j}(i, k)$, resulting in a positive core and outer negative ring. This isolates structures on a given scale, and allows the background flux to be completely subtracted (Vikhlinin et al. 1998). Full details of the wavelet transforms used are given in Henry, Finoguenov \& Briel (2004). Similarly to Finoguenov et al. $(2009,2010,2015)$ point sources in the XMM images are detected using the scales of 8 and 16 arcsec down to a wavelet significance of $4 \sigma$. The full flux of each detected point source is reconstructed using the XMM PSF model and removed from the image, following Finoguenov et al. (2009).

Having removed point sources, we apply the extended source search algorithm, applying the wavelet detection at 32- and 64-arcsec scales, and generating a noise map corresponding to the 32 -arcsec scale against which the extended flux in the reconstructed image is tested for significance. We consider extended sources of $\mathrm{X}$-ray emission as those detected above a $4 \sigma$ threshold in the wavelet analysis, relative to the level of background fluctuations. The primary target of the observations (LoCuSS clusters) has been automatically detected as a part of this procedure. The flux measurements of each detected source are made within elliptical apertures using SEXTRACTOR. SEXTRACTOR provides a description of the detected image in terms of its ellipticity, but also attempts to make a statistical fit to the data. In order to provide an ellipse in correspondence to a wavelet image, we simply boosted the significance of the image, thus forcing SExTRACTOR to provide an exact fit to the image. The extent of the emission is traced by the wavelet routine down to $1.6 \sigma$. At the depths of these $X M M$ observations, the actual signal-to-noise ratio (SNR) of the detected sources extend down to $\sim 2 \sigma$, as both the contribution of the source flux to the noise is non-negligible and the flux extraction extends to areas where the source significance is just $1.6 \sigma$ over the background level. A side-effect of the X-ray point source removal process is that the flux from any cool core in a group will also likely be lost as they are mostly unresolved.

\subsection{Determination of $\mathrm{X}$-ray group redshifts}

The optical counterparts of extended X-ray sources were identified using the combination of deep optical imaging and extensive spectroscopic data (ACReS), for which we have complete coverage over all $23 X M M$ fields. Each cluster was observed with SuprimeCam $\left(34 \times 27 \operatorname{arcmin}^{2}\right.$ field of view $)$ on the 8.2-m Subaru telescope to perform the weak lensing analysis of Okabe et al. (2010, 2013, 2016). Typical observations consisted of two bands $(V, i)$ with 30-40 min exposure times and FWHM $\sim 0.7$ arcsec, providing high-quality photometry down to $i_{A B} \sim 26$. ACReS (Haines et al. 2013, 2015) observed all 23 clusters in our sample with Hectospec, a 300-fibre multiobject spectrograph with a $1^{\circ}$ diameter circular field of view that is installed on the 6.5-m MMT telescope. Target galaxies were primarily $K$-band selected down to a limit of $m_{K}^{*}\left(z_{\mathrm{cl}}\right)+2.0$ to produce an approximately stellar mass-limited sample down to $\mathcal{M} \sim 1.5 \times 10^{10} \mathrm{M}_{\odot}$, with a $J-K$ colour selection used to efficiently target galaxies at approximately the redshift of the primary cluster (Haines et al. 2009a,b, 2013), irrespective of their star formation history (i.e. with no bias towards red sequence or star-forming galaxies). We achieve spectroscopic completeness levels of $\sim 80$ per cent for $M_{K}<-23.10\left(M_{K}^{*}+1.5\right)$ cluster galaxies within the $23 X M M$ fields.

For each X-ray source, contours of the extended X-ray emission are overlaid on the Subaru optical images. In many cases, there is a clear dominant early-type galaxy located near the centre of the $\mathrm{X}$-ray emission, which we take to be the central group galaxy. We have a spectroscopic redshift of this galaxy for all the candidate $z \lesssim 0.4$ groups. Further group members are then sought as fainter galaxies with redshifts within $1000 \mathrm{~km} \mathrm{~s}^{-1}$ of the central galaxy, located within 2-3 arcmin. In cases where no dominant central galaxy is visible, we seek at least two galaxies within 1-2 arcmin of the $\mathrm{X}$-ray centre with redshifts within $1000 \mathrm{~km} \mathrm{~s}^{-1}$ of each other. The group redshift is taken to be the mean redshift of its member galaxies. More details of the process to determine group members is given in Bianconi et al. (2018).

We are able to identify every single extended X-ray source detected at SNR $>6.0$ as a galaxy group with known redshift, comprising $32 \mathrm{X}$-ray groups at redshifts $0.06-0.67$ in addition to the targeted primary clusters. At lower significance levels (mostly at $<3 \sigma$ ), we find a number of the 'extended' X-ray sources to be centred on background QSOs, some of which had been previously identified as X-ray point sources in Chandra imaging (Haines et al. 2012). Most of these QSOs had spectra having been observed as part of ACReS strategy to target all mid-infrared bright sources $\left(f_{24}>1 \mathrm{mJy}\right)$, including those unresolved in our $K$-band imaging, resulting in a $24 \mu \mathrm{m}$-selected sample of QSOs (Xu et al. 2015a,b).

As the significance of the extended X-ray source declines, the fraction for which we find no likely counterpart in the optical images starts to increase until for $2.5<\mathrm{SNR}<3.0 \sim 40$ per cent of $\mathrm{X}$-ray sources remain unidentified. The bulk of these unidentified sources are likely compact groups of low-luminsity AGN (Finoguenov et al. 2007), which are not detected on the small (8-16 arcsec) scales. There are no galaxies that satisfy the ACReS target selection criteria within 1 arcmin of any of these unidentified X-ray sources. In other words, we do not expect to have missed identifying any $z<0.3$ groups due to incomplete spectroscopy.

Over the $23 X M M$ fields, excluding the primary clusters, we identify a total of $91 \mathrm{X}$-ray groups above an SNR limit of 3.0, with redshifts in the range $0.02-0.67$. A total of 90 of these have at least one member with a spectroscopic redshift. The remaining X-ray source is centred on a compact clump of red galaxies with $J-K$ colours consistent with the group being at $z \sim 0.6$. Fig. 1 shows the distribution of these 90 groups in the $L_{X}$ versus redshift plane. $\mathrm{X}$-ray groups that are at the same redshift as the central cluster in their $X M M$ field are indicated in red, and can be typically detected down to $L_{\mathrm{X}}(0.1-2.4 \mathrm{keV}) \sim 2 \times 10^{42} \mathrm{erg} \mathrm{s}^{-1}$.

\subsection{Estimation of the X-ray group masses}

Using the knowledge of the group's redshift, the global properties $\left(L_{X}\right)$ of the groups are determined based on the detected flux within the aperture and estimating the total flux, based on the correspondence between the fraction of the $r_{500}$ covered by the aperture and the group surface brightness profile, as described in Finoguenov et al. (2007). For most groups, the detection of their X-ray emission extends to $r_{500}$ and no correction for the aperture was made, or the applied corrections were minimal. This allows us to link the observed properties of these groups, to groups detected at similar depths in the COSMOS field (Finoguenov et al. 2007; Scoville et al. 2007) and whose X-ray luminosities were calculated in exactly the same fashion (including the excising of unresolved cool cores). These COSMOS X-ray groups were binned by $L_{\mathrm{X}}$ and redshift, and average total halo masses $\left(M_{200}\right)$ derived by Leauthaud et al. (2010) for the sub-samples by stacked weak gravitational lensing, producing a $M_{200}-L_{\mathrm{X}}$ scaling relation well described by a single power law. 


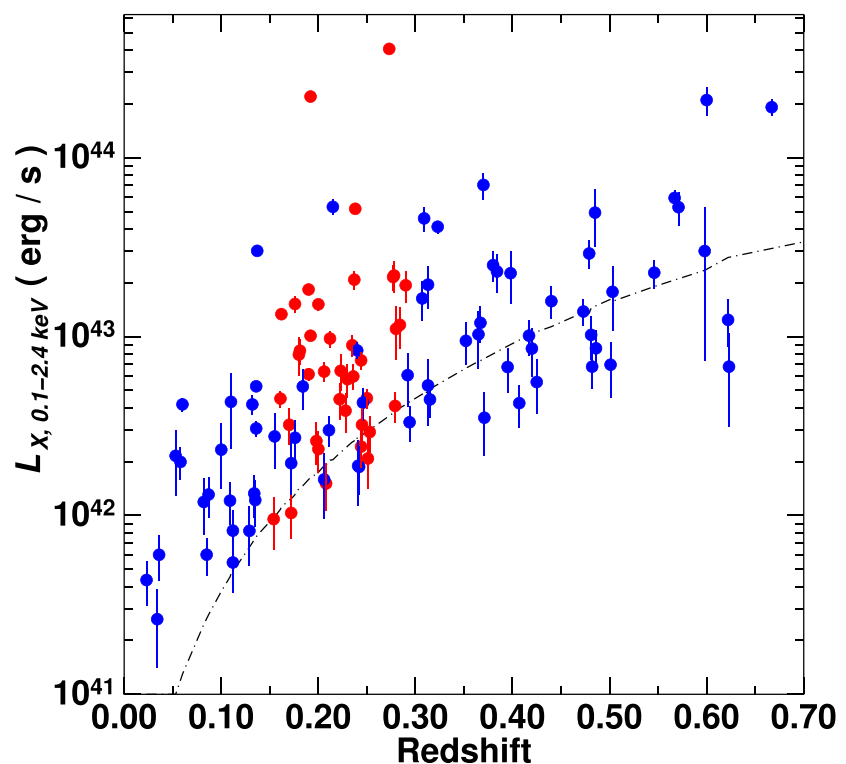

Figure 1. X-ray luminosity-redshift sampling of all the galaxy groups detected in the $X M M$ images with a confirmed spectroscopic redshift. Those galaxy groups at the redshift of the primary cluster in the same $X M M$ image are indicated in red, while blue points mark the remaining 'isolated' galaxy groups. The dot-dashed curve indicates a flux threshold of $10^{-14} \mathrm{erg} \mathrm{s}^{-1} \mathrm{~cm}^{-2}$

We estimate the total $M_{200}$ masses of our X-ray groups using the $M_{200}-L_{\mathrm{X}}$ relation of Leauthaud et al. (2010) derived by performing a joint fit between the stacked COSMOS X-ray groups and 10 high-mass clusters from LoCuSS with analogous X-ray and weaklensing data (all 10 are within our sample of 23). The resulting single power-law relation

$$
\frac{\left\langle M_{200} E(z)\right\rangle}{M_{0}}=A\left(\frac{\left\langle L_{X} E(z)^{-1}\right\rangle}{L_{X, 0}}\right)^{\alpha},
$$

with $M_{0}=10^{13.70} h_{72}^{-1} \mathrm{M}_{\odot}, L_{\mathrm{X}, 0}=10^{42.70} h_{72}^{-2} \mathrm{erg} \mathrm{s}^{-1}$, power-law index $\alpha=0.64 \pm 0.03$ and $\log _{10}(A)=0.03 \pm 0.06$, holds over two decades in mass, $M_{200} \sim 10^{13.5}-10^{15.5} \mathrm{M}_{\odot}$. Allevato et al. (2012) find that the observed bias of these COSMOS X-ray groups as measured through the projected auto-correlation function is consistent with that predicted from the group masses derived via the above relation. This relation produces group masses that are always within 20 percent of those resulting from the $M-L_{\mathrm{X}}$ scaling relation of Lovisari, Reiprich \& Schellenberger (2015), whose XMM-Newton analysis derived total masses for 20 local groups assuming hydrostatic equilibrium. The mass scatter at fixed $L_{\mathrm{X}}$ is expected to be 0.15 dex (Maughan 2007; Kettula et al. 2015; Lovisari et al. 2015). When the halo MF has a negative power law, $\mathrm{d} N / \mathrm{d} M \propto M^{-\gamma}$, then the mass scatter $\sigma_{M}$ will introduce a Malmquist bias in the masses, as more low-mass haloes are scattered up into a mass bin than high-mass haloes scattered down. For a power-law slope of $\gamma=2.5$ and mass scatter $\sigma_{M}=0.15 \mathrm{dex}$, the resulting mass bias is $\sigma_{M}^{2}(\gamma-1) \ln (10)=0.078 \mathrm{dex}$ (Leauthaud et al. 2010; Evrard et al. 2014; Rozo et al. 2014).

\subsection{A catalogue of $X$-ray groups falling into massive clusters}

X-ray groups that are infalling into the primary clusters are identified from their projected cluster-centric radii and redshifts as those located within the 'trumpet'-shaped caustic profile enclosing those galaxies identified as cluster members by Haines et al. (2013). In total, 39 of our X-ray groups were identified as being within the caustics of the primary cluster. The full list of infalling X-ray groups is presented in Table 2, including their positions, redshifts, X-ray luminosities, mass estimates, whether they have an obvious central dominant galaxy (brightest group galaxy or BGG) or not, and the number of spectroscopically confirmed members. These groups all have at least one spectroscopic member, and indeed 31/39 have $\geq 7$ members. The median number of spectroscopic members is nine. The velocity dispersions of these groups, as estimated using the gapper method (Beers, Flynn \& Gebhardt 1990), lie in the range $150-650 \mathrm{~km} \mathrm{~s}^{-1}$.

Figs 2-5 show the $0.5-2.0 \mathrm{keV}$ band X-ray images of Abell 1763, Abell 1835, Abell 963, and Abell 1758N, smoothed with a Gaussian kernel of width 12 arcsec after removing point sources. These enable the extended X-ray emission from groups in the outskirts of the cluster and in the background to be identified. Each X-ray detected group with confirmed redshift is labelled along with its $M_{200}$ mass estimate.

The X-ray emission from Abell 1763 extends significantly in the west-south-west direction (as seen previously by Zhang et al. 2007, Fig. B.6), including two sub-peaks that are identified as $\sim 10^{14} \mathrm{M}_{\odot}$ groups. Both of these peaks are close to extremely massive passive galaxies with $\mathcal{M} \sim 10^{11.6} \mathrm{M}_{\odot}$, featureless bulges and the extended diffuse envelopes characteristic of BCGs. Two more groups with masses $5-7 \times 10^{13} \mathrm{M}_{\odot}$ lie along the same axis, but in the opposite direction (east-north-east), notably directly towards Abell 1770, some $13 \mathrm{Mpc}$ distant, and within the filament of star-forming galaxies previously detected as feeding Abell 1763 along this axis (Fadda et al. 2008; Edwards et al. 2010). Again, the centres of X-ray emission from both groups are located close to massive passive galaxies.

Abell 1835 is a classic relaxed cool-core cluster, as demonstrated by its regular circular surface brightness contours, and is the most luminous cluster in the ROSAT Brightest Cluster Sample (Ebeling et al. 1998). At larger radii $\left(0.5-1.0 r_{200}\right)$, four X-ray groups are identified with masses $2.7-6.1 \times 10^{13} \mathrm{M}_{\odot}$. The two most massive groups were previously identified in the XMM data by Pereira et al. (2010) and in Chandra data by Bonamente et al. (2013), who measured temperatures of 2.7 and $2.1 \mathrm{keV}$ for the pair. The X-ray peaks are centred on massive passive galaxies with $\mathcal{M} \sim 10^{11.3} \mathrm{M}_{\odot}$. The finding of numerous groups in the infall regions of A1835 can explain its velocity dispersion profile that remains flat at $1500 \mathrm{~km} \mathrm{~s}^{-1}$ out to $2 \mathrm{Mpc}$ and significant substructures detected by the DresslerShectman test (Czoske 2004). It also demonstrates how the core of a cluster may behave as a relaxed system (Smith et al. 2005), while on larger scales it is strongly disturbed due to the presence of infalling groups.

Abell 963 has been classified as a relaxed cluster based on the joint HST strong-lensing and X-ray analysis of Smith et al. (2005), although it lacks the strong cool core of A1835. The XMM maps again reveal significant sub-structure on large scales, with three infalling groups identified. While two are relatively poor systems $\left(M_{200} \sim 2-3 \times 10^{13} \mathrm{M}_{\odot}\right)$, the third is a massive group with $M_{200}=1.0 \times 10^{14} \mathrm{M}_{\odot}$, located at a projected distance of $1.0 r_{200}$ from A963. A Subaru $I_{C}$ image centred on the group is shown in Fig. 6, with the known group members labelled. The X-ray emission (magenta contours) is centred on a massive passive galaxy $\left(\mathcal{M} \sim 10^{11.2} \mathrm{M}_{\odot}\right)$ that dominates the group. The BUDHIES team carried out an ultra-deep H I survey of Abell 963 and its environs, revealing that galaxies within the most massive $\mathrm{X}$-ray group are strongly deficient in $\mathrm{H}_{\mathrm{I}}$, relative to the lower mass groups and the 
Table 2. The $X M M$ sample of infalling galaxy groups. Columns $(1,2)$ : IAU name and short ID of X-ray group. Columns $(3,4)$ : coordinates of the centroid of the group's X-ray emission $(\alpha, \delta)$. Column (5) mean redshift of group members. Column (6): rest-frame X-ray luminosity of the group in the 0.1-2.4 keV band. Column (7): estimate of the cluster mass $M_{200}$ in units of $10^{13} \mathrm{M}_{\odot}$. Column (8): SNR of the X-ray detection. Column (9): projected distance of group from the primary cluster centre in units of $r_{200}$. Column (10) flag indicating whether the group contains a dominant central galaxy (BGG) or not. Column (11): number of spectroscopically confirmed group members.

\begin{tabular}{|c|c|c|c|c|c|c|c|c|c|c|}
\hline $\begin{array}{l}\text { IAU name } \\
\text { XMMU }\end{array}$ & $\begin{array}{l}\text { Group } \\
\text { ID }\end{array}$ & $\begin{array}{c}\text { Right } \\
\text { Ascension } \\
(\mathrm{J} 2000)\end{array}$ & $\begin{array}{l}\text { Declination } \\
\text { (J2000) }\end{array}$ & $\langle z\rangle$ & $\begin{array}{c}L_{\mathrm{X}} \\
(0.1-2.4 \mathrm{keV}) \\
\left(10^{42} \mathrm{erg} \mathrm{s}^{-1}\right)\end{array}$ & $\begin{array}{c}M_{200} \\
\left(10^{13} \mathrm{M}_{\odot}\right)\end{array}$ & SNR & $\frac{r_{\text {proj }}}{r_{200}}$ & $\begin{array}{l}\mathrm{BGG} \\
\mathrm{Y} / \mathrm{N}\end{array}$ & $N_{z}$ \\
\hline J005611.3+262445 & A115-g6 & $00: 56: 11.33$ & $+26: 24: 45.8$ & 0.19326 & $10.11 \pm 0.51$ & $7.77 \pm 1.15$ & 19.8 & 0.623 & Y & 5 \\
\hline J005601.5+262727 & A115-g7 & 00:56:01.52 & $+26: 27: 27.6$ & 0.18919 & $6.16 \pm 0.48$ & $5.67 \pm 0.83$ & 12.9 & 0.502 & Y & 13 \\
\hline J005616.1+262259 & A115-g8 & $00: 56: 16.13$ & $+26: 22: 59.0$ & 0.19005 & $18.31 \pm 0.58$ & $11.38 \pm 1.68$ & 31.7 & 0.797 & $\mathrm{Y}$ & 7 \\
\hline J013205.1-133953 & A209-g6 & 01:32:05.17 & $-13: 39: 53.4$ & 0.21212 & $9.74 \pm 1.00$ & $7.47 \pm 1.17$ & 9.7 & 0.468 & Y & 12 \\
\hline J013137.0-134501 & A209-g10 & 01:31:37.04 & $-13: 45: 01.9$ & 0.20034 & $2.35 \pm 0.62$ & $3.03 \pm 0.68$ & 3.8 & 1.020 & Y & 2 \\
\hline J080125.6+360537 & A611-g5 & 08:01:25.69 & $+36: 05: 37.0$ & 0.28389 & $11.62 \pm 3.04$ & $7.88 \pm 1.73$ & 3.8 & 0.845 & $\mathrm{Y}$ & 10 \\
\hline J080111.0+360521 & A611-g6 & 08:01:11.04 & $+36: 05: 21.8$ & 0.28918 & $19.34 \pm 3.81$ & $10.86 \pm 2.12$ & 5.1 & 0.472 & Y & 7 \\
\hline J083042.5+655829 & A665-g2 & $08: 30: 42.55$ & $+65: 58: 29.8$ & 0.18180 & $8.33 \pm 1.34$ & $6.92 \pm 1.22$ & 6.2 & 0.623 & Y & 9 \\
\hline J084300.7+362841 & A697-g4 & 08:43:00.79 & $+36: 28: 41.3$ & 0.27985 & $10.40 \pm 3.66$ & $7.65 \pm 2.02$ & 3.0 & 0.714 & $\mathrm{~N}$ & 11 \\
\hline $\mathrm{J} 101708.2+385058$ & A963-g1 & $10: 17: 08.24$ & $+38: 50: 58.1$ & 0.19898 & $2.61 \pm 0.70$ & $3.25 \pm 0.73$ & 3.8 & 1.293 & $\mathrm{Y}$ & 9 \\
\hline $\mathrm{J} 101635.4+391005$ & A963-g5 & $10: 16: 35.43$ & $+39: 10: 05: 7$ & 0.20915 & $1.51 \pm 0.45$ & $2.27 \pm 0.57$ & 3.3 & 0.989 & $\mathrm{~N}$ & 7 \\
\hline $\mathrm{J} 101640.1+385443$ & A963-g10 & $10: 16: 40.13$ & $+38: 54: 43.0$ & 0.20129 & $15.14 \pm 1.12$ & $10.00 \pm 1.52$ & 13.5 & 1.012 & Y & 21 \\
\hline $\mathrm{J} 133210.6+503031$ & A1758-g7 & $13: 32: 10.67$ & $+50: 30: 31.5$ & 0.27903 & $4.09 \pm 0.80$ & $4.06 \pm 0.77$ & 5.1 & 0.643 & $\mathrm{Y}$ & 18 \\
\hline $\mathrm{J} 133231.4+502437$ & A1758-g8 & $13: 32: 31.43$ & $+50: 24: 37.1$ & 0.27244 & $405.61 \pm 4.38$ & $77.24 \pm 15.0$ & 92.7 & 0.922 & Y & 16 \\
\hline $\mathrm{J} 133535.0+410602$ & A1763-g5 & $13: 35: 35.04$ & $+41: 06: 02.4$ & 0.23572 & $5.99 \pm 0.99$ & $5.36 \pm 0.94$ & 6.0 & 0.706 & $\mathrm{Y}$ & 11 \\
\hline $\mathrm{J} 133612.2+410455$ & A1763-g6 & $13: 36: 12.27$ & $+41: 04: 55.3$ & 0.23591 & $8.94 \pm 1.23$ & $6.94 \pm 1.16$ & 7.3 & 1.166 & $\mathrm{Y}$ & 7 \\
\hline $\mathrm{J} 133452.7+405702$ & A1763-g7 & $13: 34: 52.77$ & $+40: 57: 02.6$ & 0.23725 & $51.80 \pm 2.28$ & $21.30 \pm 3.40$ & 22.7 & 0.574 & $\mathrm{Y}$ & 17 \\
\hline $\mathrm{J} 133505.4+405829$ & A1763-g9 & $13: 35: 05.44$ & $+40: 58: 29.7$ & 0.23703 & $20.80 \pm 2.44$ & $11.89 \pm 1.97$ & 8.5 & 0.287 & $\mathrm{Y}$ & 11 \\
\hline $\mathrm{J} 133533.3+405208$ & A1763-g11 & $13: 35: 33.39$ & $+40: 52: 08.1$ & 0.22929 & $3.85 \pm 0.95$ & $4.07 \pm 0.89$ & 4.0 & 0.856 & Y & 4 \\
\hline $\mathrm{J} 164010.8+464851$ & A2219-g5 & $16: 40: 10.88$ & $+46: 48: 51.4$ & 0.23548 & $5.77 \pm 1.22$ & $5.26 \pm 1.03$ & 4.7 & 0.602 & $\mathrm{Y}$ & 1 \\
\hline $\mathrm{J} 164007.1+463629$ & A2219-g7 & $16: 40: 07.17$ & $+46: 36: 29.5$ & 0.22214 & $6.43 \pm 1.47$ & $5.57 \pm 1.13$ & 4.4 & 0.571 & $\mathrm{~N}$ & 5 \\
\hline $\mathrm{J} 215309.4+174224$ & A2390-g1 & 21:53:09.47 & $+17: 42: 24.9$ & 0.22184 & $4.46 \pm 1.04$ & $4.49 \pm 0.93$ & 4.3 & 0.584 & Y & 1 \\
\hline $\mathrm{J} 171926.0+263333$ & $\mathrm{R} 1720-\mathrm{g} 4$ & $17: 19: 26.09$ & $+26: 33: 33.0$ & 0.15380 & $0.95 \pm 0.31$ & $1.77 \pm 0.46$ & 3.1 & 0.939 & $\mathrm{Y}$ & 1 \\
\hline $\mathrm{J} 172002.2+262944$ & R1720-g6 & $17: 20: 02.23$ & $+26: 29: 44.8$ & 0.16144 & $4.50 \pm 0.46$ & $4.74 \pm 0.74$ & 9.6 & 0.705 & $\mathrm{~N}$ & 10 \\
\hline $\mathrm{J} 145741.1+222333$ & Z7160-g5 & $14: 57: 41.14$ & $+22: 23: 33.2$ & 0.25349 & $2.92 \pm 0.67$ & $3.34 \pm 0.68$ & 4.4 & 1.006 & $\mathrm{~N}$ & 2 \\
\hline
\end{tabular}

infall regions of the cluster, and providing evidence of the impact of pre-processing on galaxies in infalling groups, stripping their gas contents and quenching star formation before they are accreted into the cluster (Jaffé et al. 2016).

Abell 1758 is a well-known double cluster system with two distinct clusters (A1758N and A1758S) separated by 8 arcmin (Rizza et al. 1998) in the plane of the sky (or $2 \mathrm{Mpc}$ at $z=0.279$ ). Both A1758N and A1758S are undergoing major mergers, with Chandra imaging of $\mathrm{A} 1758 \mathrm{~N}$ revealing two remnant cores separated by $800 \mathrm{kpc}$ and shock fronts (David \& Kempner 2004). Our XMM analysis has revealed a further sub-structure within this complex system, a $4 \times 10^{13} \mathrm{M}_{\odot}$ group that lies $6 \operatorname{arcmin}(1.5 \mathrm{Mpc})$ ESE from $\mathrm{A} 1758 \mathrm{~N}$ and at the same redshift $(z=0.279)$. The X-ray emission is centred on a compact clump of four group members, including the BCG (Fig. 7), with many more group galaxies in the vicinity.

Figs 8 and 9 show two examples of our poorest infalling X-ray groups: A209-g10 and A2390-g1. In the former, the X-ray emission is centred on an obvious dominant group galaxy $(\mathcal{M} \sim$ $10^{11.2} \mathrm{M}_{\odot}$ ), with a nearby second bright galaxy within $400 \mathrm{~km} \mathrm{~s}^{-1}$. In the latter, there is only one bright galaxy located within the $\mathrm{X}$-ray contours, a massive $\left(\mathcal{M} \sim 10^{11.0} \mathrm{M}_{\odot}\right)$ passive galaxy. In 


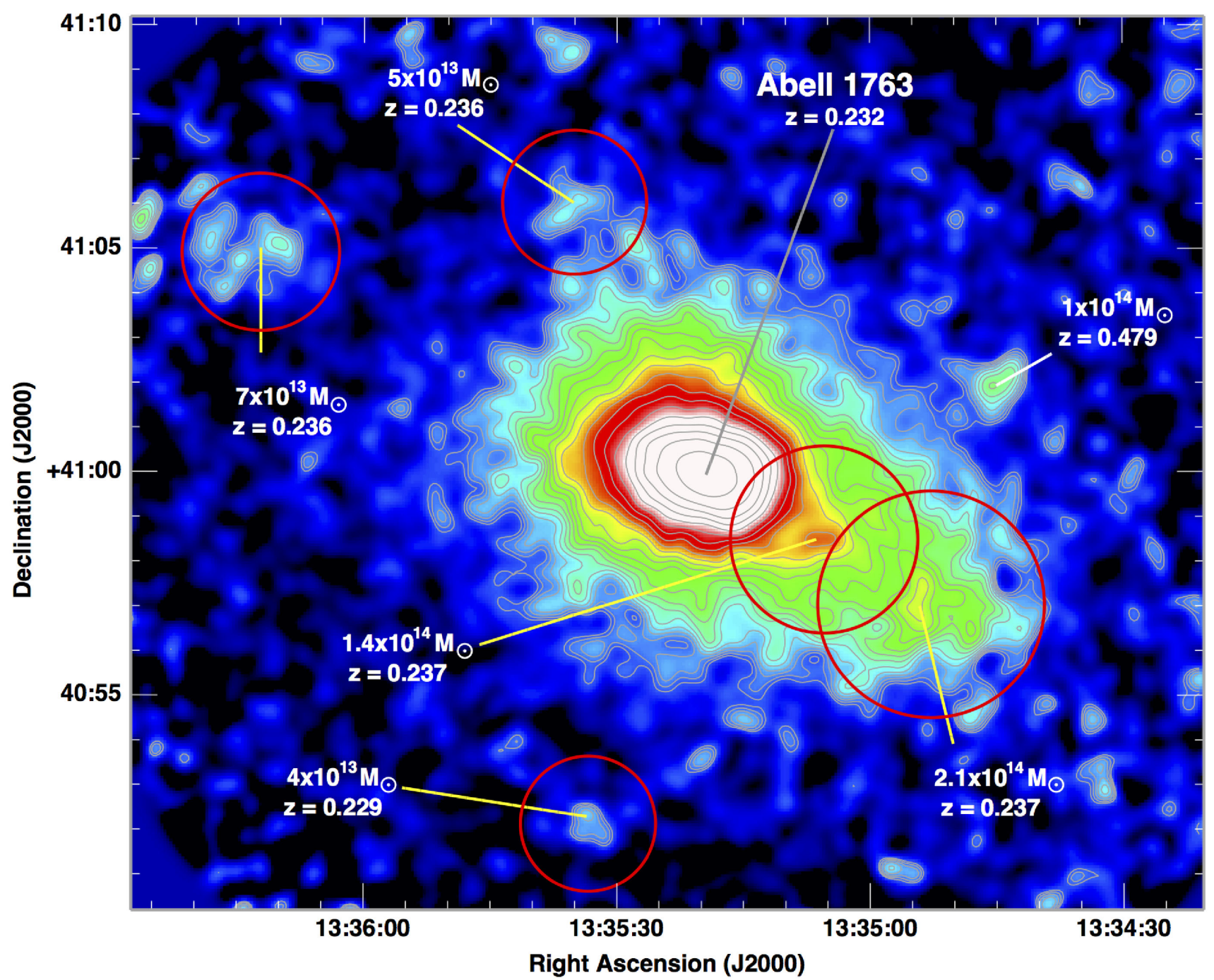

Figure 2. Extended X-ray emission from Abell 1763. All spectroscopically confirmed X-ray groups are indicated and labelled by their redshift and estimated $M_{200}$ value. Those groups that are infalling into Abell 1763 are marked by red circles of diameter $r_{200}$.

both cases, the association of the X-ray emission with the group galaxies appears robust and unambigious.

\subsection{A comparison sample of galaxy groups around clusters in the Millennium simulation}

To understand the fates of the infalling X-ray groups, estimate the mass completeness of the $X M M$ observations, and to compare our results to predictions from $\Lambda \mathrm{CDM}$ cosmological models, we have created a comparison sample of galaxy groups in the vicinity of the 75 most massive clusters $\left(M_{200}>4.0 \times 10^{14} h^{-1} \mathrm{M}_{\odot}\right.$ at $\left.z=0.0\right)$ from the Millennium simulation (MS; Springel et al. 2005), a cosmological DM simulation covering a $\left(500 h^{-1} \mathrm{Mpc}\right)^{3}$ volume. Following Haines et al. (2015), we have extracted DM haloes with $M_{200}>10^{13} h^{-1} \mathrm{M}_{\odot}$ from the MPA Halo (MHalo) catalogue within $20 \times 20 \times 140 h^{-3} \mathrm{Mpc}^{3}$ volumes centred on each cluster. The periodic nature of the simulation cube allows the extracted volume to always be centred on the target halo. These volumes are extended in the $z$-direction so that, for a distant observer viewing along this axis, all galaxy groups with LOS velocities within $5000 \mathrm{~km} \mathrm{~s}^{-1}$ of the cluster redshift are included, enabling projection effects to be fully accounted for and quantified. The group halo positions and velocities relative to the primary cluster halo are measured at the $z=0.21$ snapshot from the simulation, and artificial observations created assuming a distant observer along the $z$-axis. Those DM haloes whose LOS velocities place them within the caustics defined by the cluster galaxy members used in Haines et al. (2015) are then retained to form our comparison sample of simulated infalling galaxy groups, and the $M_{200}-L_{\mathrm{X}}$ scaling-relation of equation (1) used to predict their X-ray luminosities (no scatter in $L_{\mathrm{X}}$ is added). Since this is a DM-only simulation, baryon back-reaction effects are not included.

The completeness of each $X M M$ image as a function of group mass is measured following the procedure outlined in Finoguenov et al. (2015), by generating simulated galaxy groups of a given mass and redshift, and the tabulation of Finoguenov et al. (2007) to predict the parameters of the beta model used to describe the surface brightness profile of their X-ray emission. Unlike Finoguenov et al. (2015), we use the simulated infalling groups from the MS to model the mass and radial distribution of groups around the 


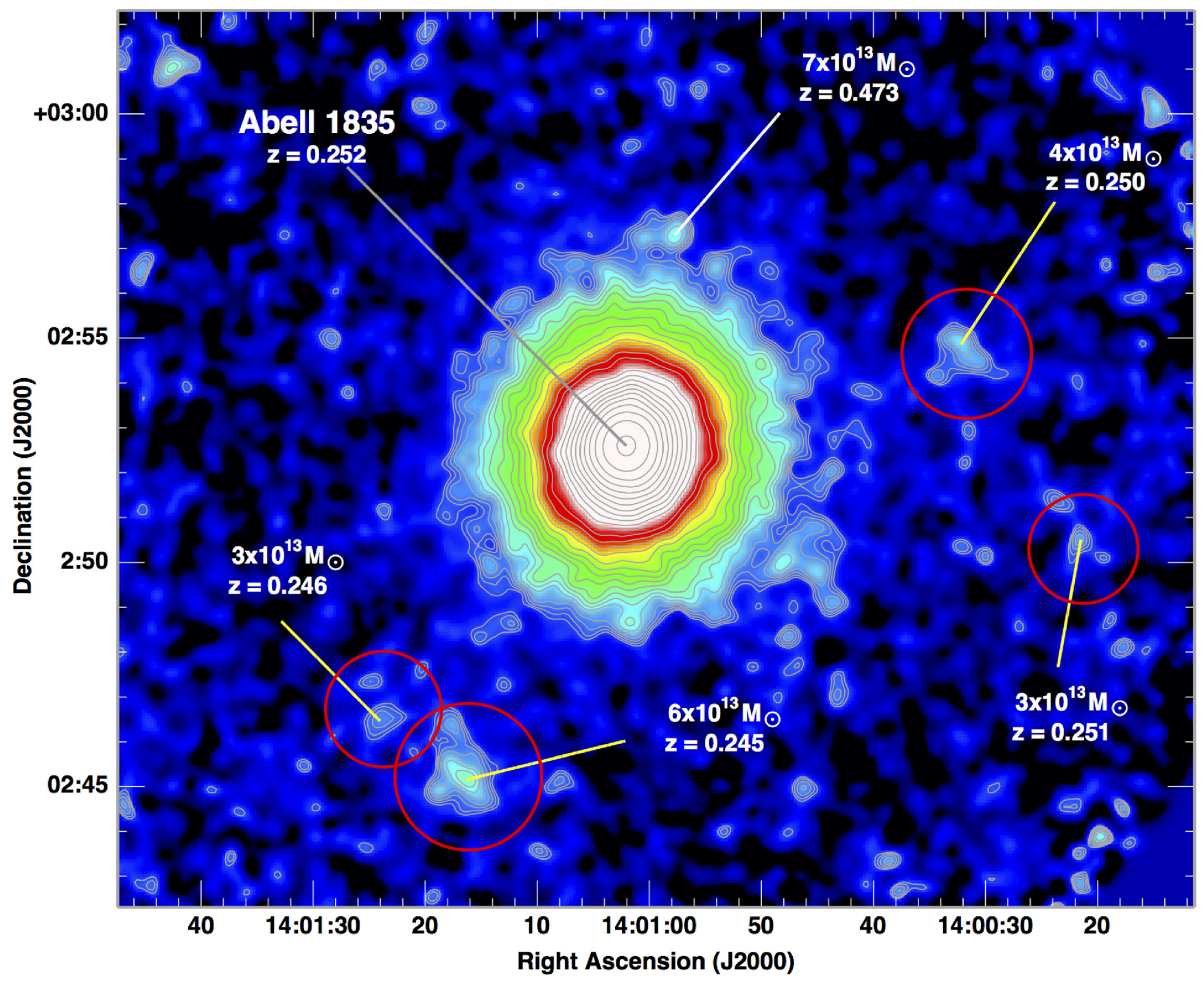

Figure 3. Extended X-ray emission from Abell 1835. All spectroscopically confirmed X-ray groups are indicated and labelled by their redshift and estimated $M_{200}$ value. Those groups that are infalling into Abell 1835 are marked by red circles of diameter $r_{200}$.

cluster, rather than assume a random spatial distribution and mass distribution defined by a $\Lambda \mathrm{CDM}$ cosmological model. This step is necessary because the distribution of groups in and around clusters is not described by linear growth theory and simulations are required. The particular choice of the cosmology in the simulations is not so important, as the abundance of subhalos is a not a very sensitive function of the cosmology (Taylor \& Babul 2005) and the subhalo MF scales well at any redshift as a function of the subhalo ratio to the total halo mass (Giocoli et al. 2008).

We take each simulated X-ray group, in turn, and place that individual group into each of the $X M M$ images, the wavelet detection applied and catalogues produced to confirm whether that simulated group was recovered. Repeating this process for all the simulated $\mathrm{X}$-ray groups, we then measure the fraction of simulated groups detected as a function of their halo mass for each $X M M$ image. Fig. 10 plots the fraction of these simulated groups recovered by the wavelet-detection algorithm as a function of group halo mass $\left(M_{200}\right.$; Fig. 10a), group-cluster mass ratio (Fig. 10b), and clustercentric radius (Fig. 10c). The blue and grey curves, respectively, show the recovery rates averaged over the 23 clusters, and for each individual $X M M$ observation. We expect to detect $\sim 70$ per cent of groups with $M_{200} \sim 5 \times 10^{13} \mathrm{M}_{\odot}$ over the $23 X M M$ fields. The recovery rate only rises slightly to higher masses, which is partly due to some of the XMM observations having high backgrounds, negatively affecting the detection rate even at masses approaching $10^{14} \mathrm{M}_{\odot}$.

The key cause of incompleteness at masses above $10^{13.5} \mathrm{M}_{\odot}$ is revealed in Fig. 10c, where the recovery rate (blue curve) plummets from $\sim 80$ per cent at $0.5<\left(r_{\text {proj }} / r_{200}\right)<1.0$ to just $\sim 20$ per cent in the cluster cores $\left(r_{\text {proj }} \lesssim 0.35 r_{200}\right)$. This is due to difficulty in distinguishing the $\mathrm{X}$-ray emission from groups from the much brighter emission from the primary cluster. This explains the notable absence of X-ray groups in the cluster core regions in our sample (Table 2; Fig. 11). This affects our overall mass completeness level, and the red dashed lines in Figs 10a,b show the improved completeness levels after excising the cluster cores $\left(r_{\text {proj }}<0.35 r_{200}\right)$.

We also start missing X-ray groups at large cluster-centric radii due to the limited field of view of the $X M M$ instruments, as shown by the black dashed curve in Fig. 10c. While the $X M M$ data provide 


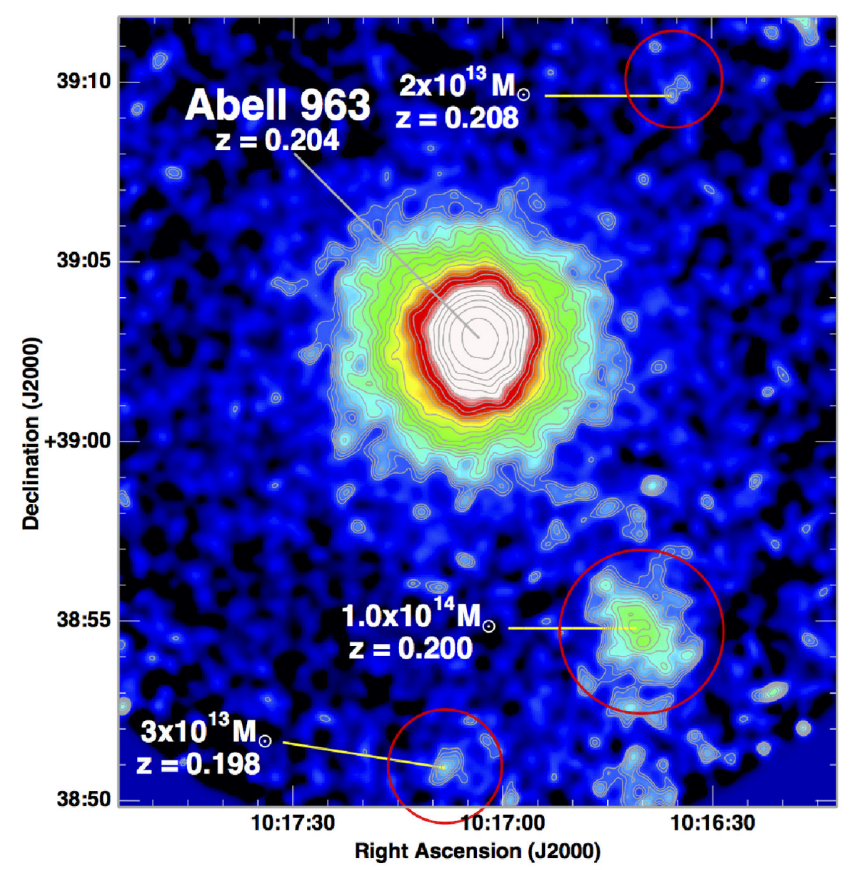

Figure 4. Extended X-ray emission from Abell 963. The three spectroscopically confirmed X-ray groups infalling into A963 are marked by red circles and labelled as in Figs 2 and 3.

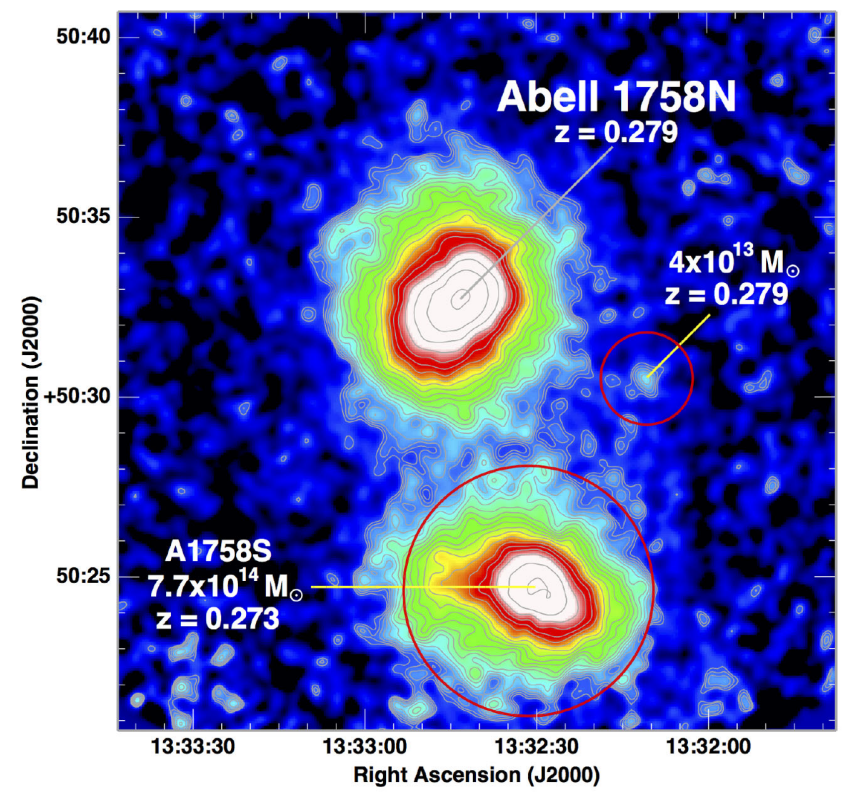

Figure 5. Extended X-ray emission from Abell 1758N. Both A1758S and the second X-ray group falling into A1758N are marked by red circles and labelled as before.

complete coverage inside $r_{200}$, the coverage fraction drops rapidly beyond $1.3 r_{200}$. Our $X M M$ data are thus most efficient at detecting infalling X-ray groups at cluster-centric distances of $0.35-1.3 r_{200}$.

\section{RESULTS}

A total of $39 \mathrm{X}$-ray groups are identified across the $23 X M M$ images as being associated with the primary clusters, down to an SNR limit of 3. Six of the clusters (A267, A291, A383, A1689, RXJ2129,

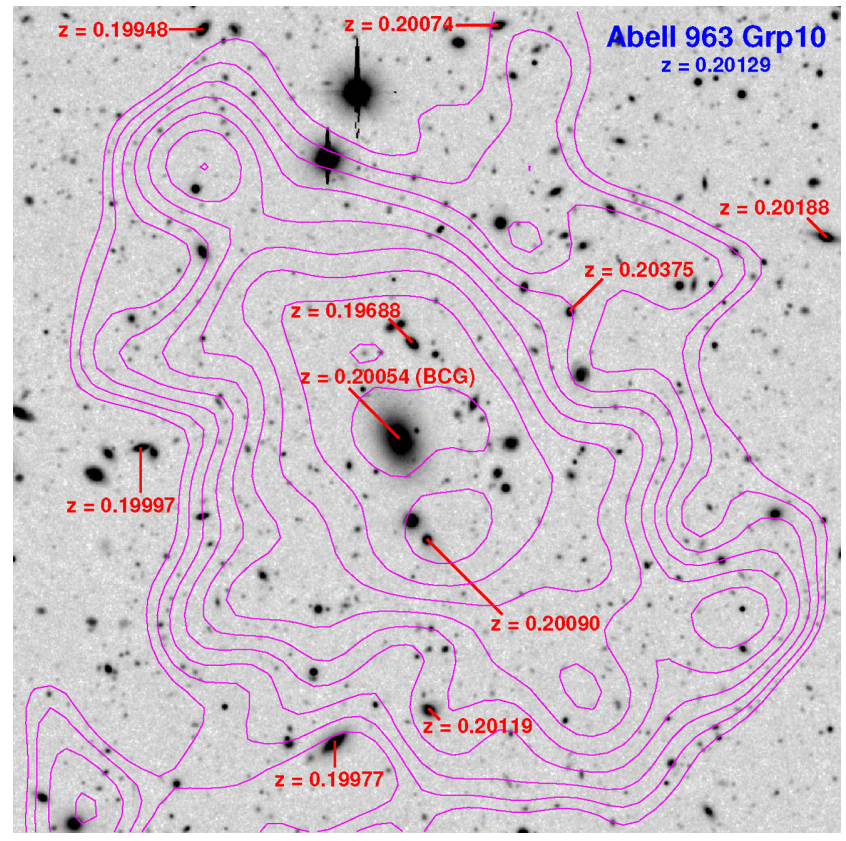

Figure 6. A $214 \times 214 \mathrm{arcsec}^{2}$ Subaru/SuprimeCAM $I_{C}$-band image centred on the infalling group A963-g10 $\left(M_{200}=1.0 \times 10^{14} \mathrm{M}_{\odot}\right)$. Magenta contours indicate the extended $\mathrm{X}$-ray emission from the group. Spectroscopic group members are indicated in red, and their redshift reported.

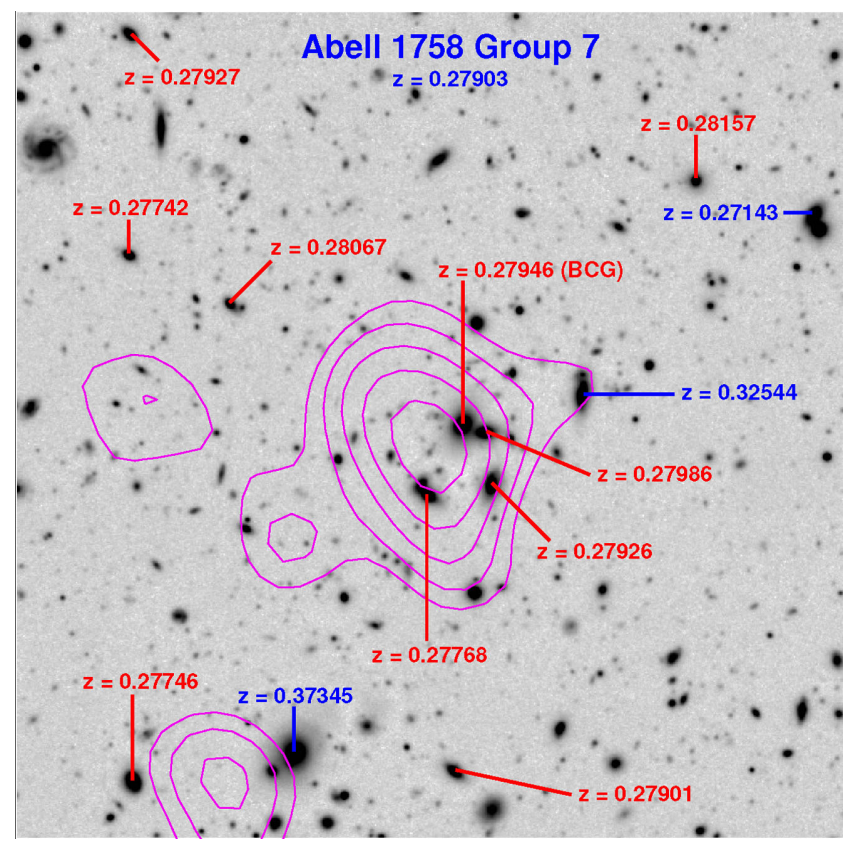

Figure 7. A $160 \times 160 \operatorname{arcsec}^{2}$ Subaru/SuprimeCAM $R_{C}$-band image centred on the infalling group A1758-g7 $\left(M_{200}=4.1 \times 10^{13} \mathrm{M}_{\odot}\right)$. Magenta contours indicate the extended X-ray emission from the group. Spectroscopic group members are indicated in red, and other galaxies with known redshifts indicated in blue.

and Z2089) have no X-ray detected groups in their infall regions, while Abell 1763 has the most with five. The numbers of groups around each cluster are consistent with the 39 groups being allocated randomly to the 23 clusters. 


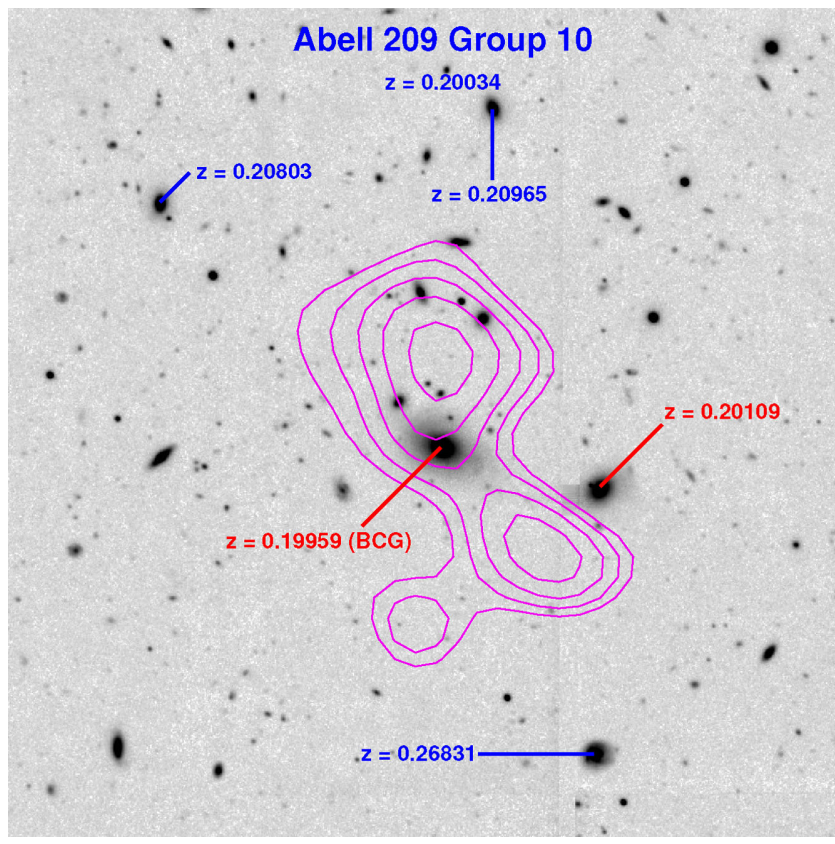

Figure 8. A $160 \times 160 \operatorname{arcsec}^{2}$ SuprimeCAM $i$-band image centred on the infalling group A209-g10 $\left(M_{200}=3.0 \times 10^{13} \mathrm{M}_{\odot}\right)$. The two group members are indicated in red. Other galaxies with known redshifts are labelled in blue.

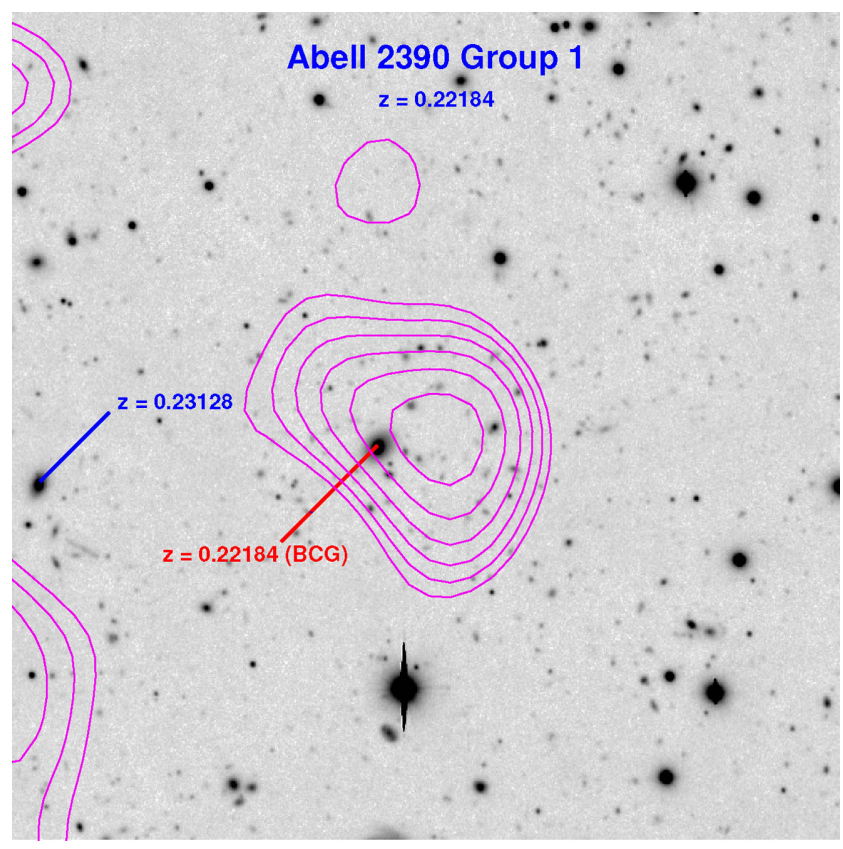

Figure 9. A $160 \times 160 \operatorname{arcsec}^{2}$ SuprimeCAM $R_{C}$-band image centred on the infalling group A2390-g1 $\left(M_{200}=4.5 \times 10^{13} \mathrm{M}_{\odot}\right)$.

\subsection{Spatial and velocity distribution of the infalling $\mathrm{X}$-ray groups}

Fig. 11 shows the distribution of the 39 X-ray groups (magenta symbols) in the stacked caustic diagram. This plots the LOS velocity of each group relative to the central redshift of the primary cluster, scaled by the velocity dispersion of all cluster members within $r_{200}$ $\left(\sigma_{v_{\mathrm{cl}}}\right.$; taken from Haines et al. 2015), against its projected clustercentric distance. This shows how all these groups lie within the 'trumpet'-shaped region formed by the galaxies (solid grey points) that have been spectroscopcially identified as members of the same 23 clusters, demonstrating that the groups are indeed associated with the clusters.

The overall distribution of relative LOS velocities for these 39 groups is shown by the histogram on the right. As discussed in detail in Haines et al. (2015), both the width and shape of the LOS velocity distribution of populations of objects in and around galaxy clusters depend strongly on when they have been (or will be) accreted into the cluster. Low LOS velocity dispersions and Gaussian distributions are indicators of virialized populations, while high LOS velocity dispersions and flat top-hat distributions are associated with objects on their first infall (Haines et al. 2015; Hikage \& Yamamoto 2016).

While the velocity dispersion of the X-ray groups about the cluster redshift is marginally lower than that of the overall cluster galaxy population, $\sigma\left(v_{\mathrm{gr}}-v_{\mathrm{cl}}\right)=0.86 \pm 0.08 \sigma_{v_{\mathrm{cl}}}$, half of the XMM groups are located along the caustics where objects on their first infall into the clusters are expected to be found. The histogram shows an excess of groups with velocities around $-1.4 \sigma_{v_{\mathrm{cl}}}$ and $+1.0 \sigma_{v_{\mathrm{cl}}}$, relative to expectations from a Gaussian distribution (blue dashed curve), and a shortfall of groups with LOS velocities around zero. The kurtosis of the group-cluster LOS velocity distribution is negative $(\gamma=$ $-0.82 \pm 0.36$ ), being inconsistent at the $2.3 \sigma$ level with that of a Gaussian distribution $(\gamma=0.0)$, and closer to the value expected for a flat top-hat distribution $(\gamma=-1.2)$.

The infalling X-ray groups are heavily concentrated within the radial range $0.35-1.3 r_{200}$ (vertical dashed lines), where the $X M M$ data are predicted to be most complete (Fig. 10c), with 37/39 groups from our sample found within this range. That is not to say that we could not detect infalling X-ray groups beyond $1.3 r_{200}$. In fact, of the 52 other X-ray groups detected by $X M M$, but with redshifts inconsistent with that of the primary cluster, 26 were found at $r_{\text {proj }}>1.3 r_{200}$.

Fig. 12 compares the surface number density $\Sigma(r)$ distributions of the 39 infalling X-ray groups (red points) and the other 52 'isolated' $\mathrm{X}$-ray groups (i.e. not in the vicinity of a massive cluster; blue points) found in the same $X M M$ fields, as a function of projected cluster-centric radius. The two radial distributions are markedly different. The infalling X-ray groups show a sharp peak at 0.5 $0.75 r_{200}$, before dropping off rapidly at larger radii and no infalling groups beyond $1.33 r_{200}$, while the other $\mathrm{X}$-ray groups in the same field show a rather flat radial distribution over $0.5-1.75 r_{200}$. In both cases, the number densities of groups drops inside $0.5 r_{200}$ and are absent within $0.25 r_{200}$, due to the inability to detect $\mathrm{X}$-ray groups projected close to the cluster core in the $X M M$ data (Fig. 10c). The upper panel shows the corresponding cumulative radial distributions of the infalling X-ray groups (red curve) and isolated 'field' groups (blue curve). The infalling groups are found much closer on average to the primary cluster than the back/foreground X-ray groups. The median projected cluster-centric distance of the infalling groups is $0.71 r_{200}$, compared to $1.29 r_{200}$ for the remaining X-ray groups detected in the same $X M M$ fields. The largest difference is seen at the virial radius, with $35 / 39$ ( 90 per cent) infalling groups having $r_{\text {proj }}<1.02 r_{200}$, while $39 / 52$ (75 per cent) of the isolated groups lie at $r_{\text {proj }}>1.02 r_{200}$. The non-parametric Mann-Whitney U-test confirms the radial distributions of the infalling and field groups to be inconsistent at the $6.0 \sigma$ level.

As these other X-ray groups are not associated with the cluster, we should expect them to be uniformly distributed across the $X M M$ images, but then be affected by the same radial selection biases as the infalling groups (Fig. 10c). The green dashed curve shows the expected cumulative radial distribution of groups 

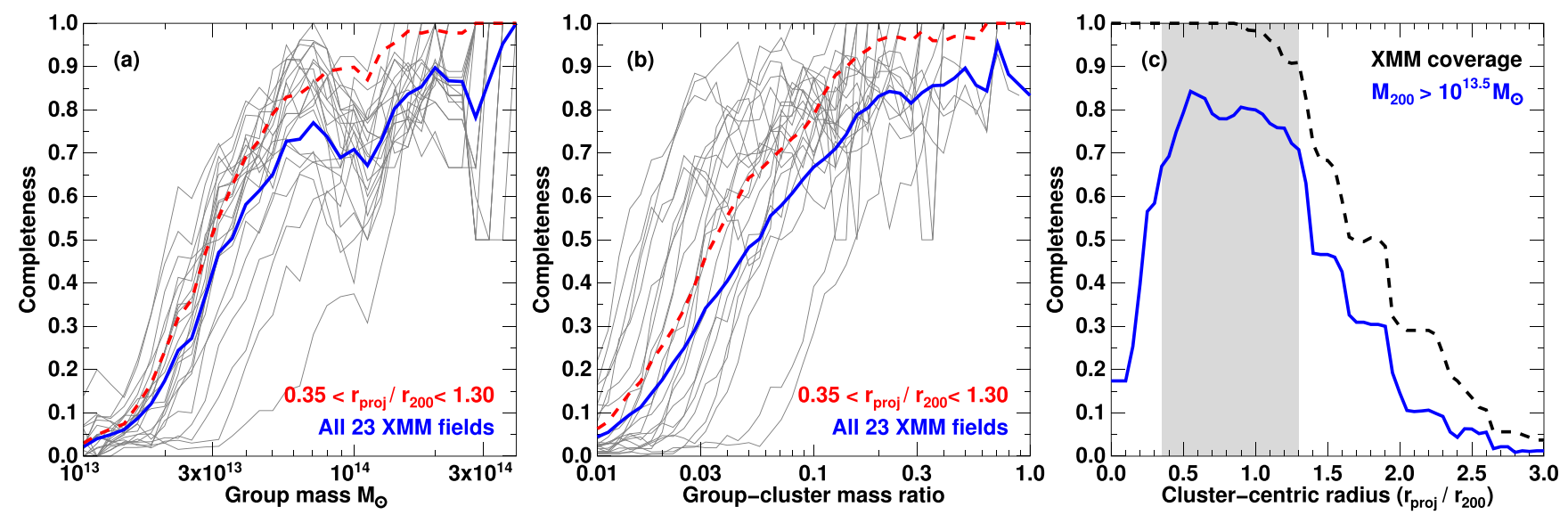

Figure 10. Fraction of simulated galaxy groups recovered by the wavelet-reconstruction detection algorithm in each of the $X M M$ images (grey lines) as a function of group $M_{200}$ mass (left-hand panel) and group-cluster mass ratio (central panel). The blue curves show the mean recovery rate averaged over all 23 clusters. The red dashed curves show the mean recovery rate after excluding the cluster core regions with $r_{\text {proj }}<0.35 r_{200}$ and the outer regions affected by vignetting $\left(r_{\text {proj }}>1.3 r_{200}\right)$. Right-hand panel: Fraction of simulated galaxy groups with $M_{200}>10^{13.5} \mathrm{M}_{\odot}$ covered by the $X M M$ imaging (black dashed curve) and recovered by the detection algorithm (blue curve) as a function of cluster-centric radius. The grey shaded region indicates the radial range over which the recoverate rate of X-ray groups within the XMM images should be highest (red dashed lines in panels a,b).

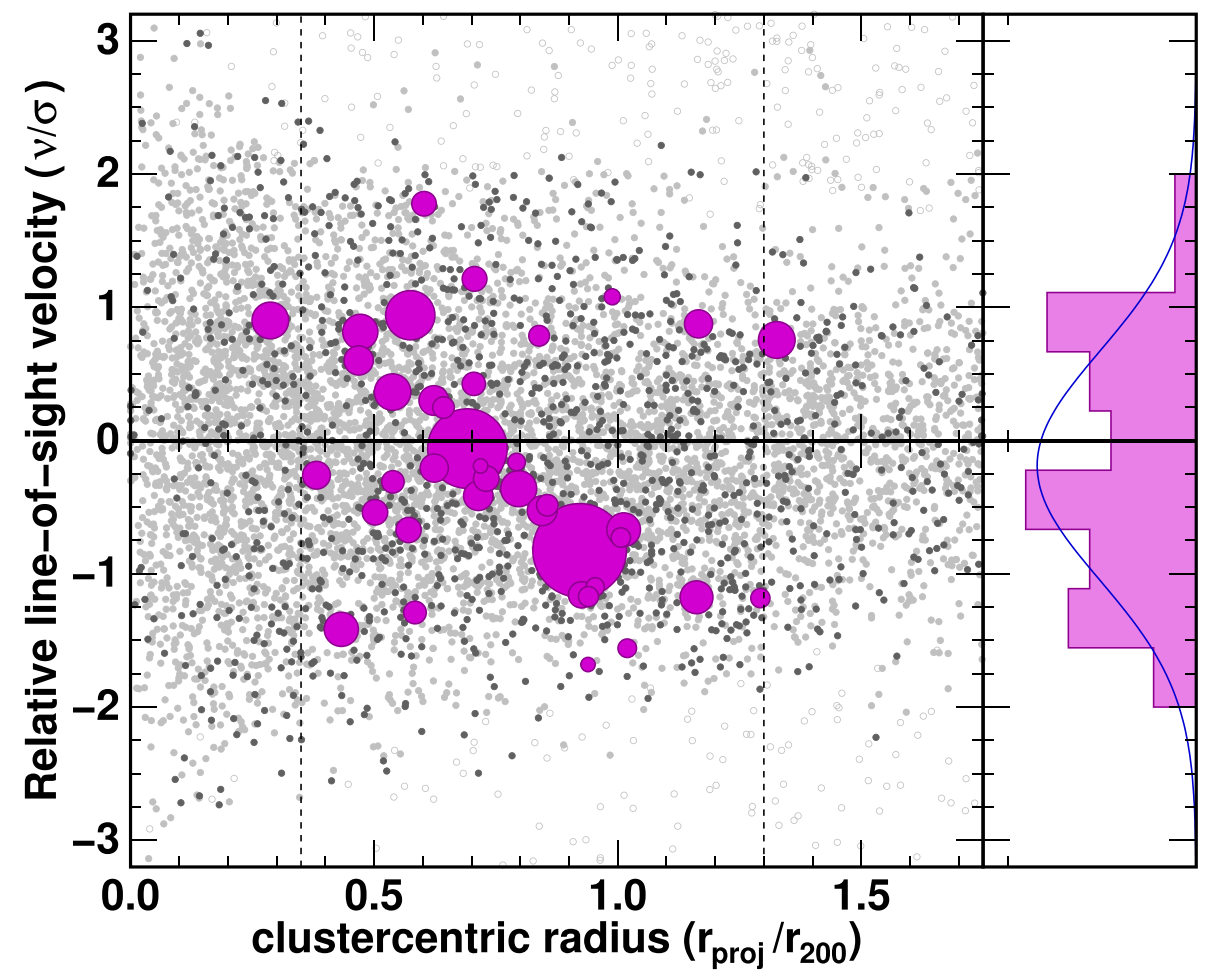

Figure 11. Stacked phase-space diagram, $\left(v_{l o s}-\langle v\rangle\right) / \sigma_{v}$ versus $r_{\text {proj }} / r_{200}$ of the $39 X M M$ groups (magenta symbols) and member galaxies (grey solid points) for all 23 clusters in our sample. The size of each symbol indicates the group mass. Darker grey symbols indicating star-forming galaxies detected at $24 \mu \mathrm{m}$. Open symbols indicate field galaxies. The vertical dashed lines enclose the $0.35 \leq\left(r_{\text {proj }} / r_{200}\right)<1.30$ region, where the $X M M$ data are most complete. The solid histogram on the right-hand side shows the distribution of relative LOS velocities for the 39 XMM groups, while the blue curve shows a Gaussian distribution with the same mean and standard deviation.

assuming a uniform spatial distribution over the $X M M$ images, and taking into account the loss of sensitivity in the cluster core regions. The fore/background X-ray groups are consistent with being randomly distributed across the $X M M$ fields, while the infalling X-ray groups are clearly not.

The strong preference of infalling X-ray groups to lie within $r_{200}$ can be understood in terms of the expected clustering of group-mass systems around massive clusters. The light-grey shaded histogram shows the surface number density of $M_{200}>10^{13} \mathrm{M}_{\odot}$ DM haloes (groups) in the vicinity of the 75 most massive clusters from the MS, as a function of projected cluster-centric distance. The surface number density shows a sharp peak inside $0.5 r_{200}$, before rapidly dropping to larger radii, falling sixfold by $r_{\text {proj }} \sim 2 r_{200}$. This is the predicted radial distribution of groups before accounting for 


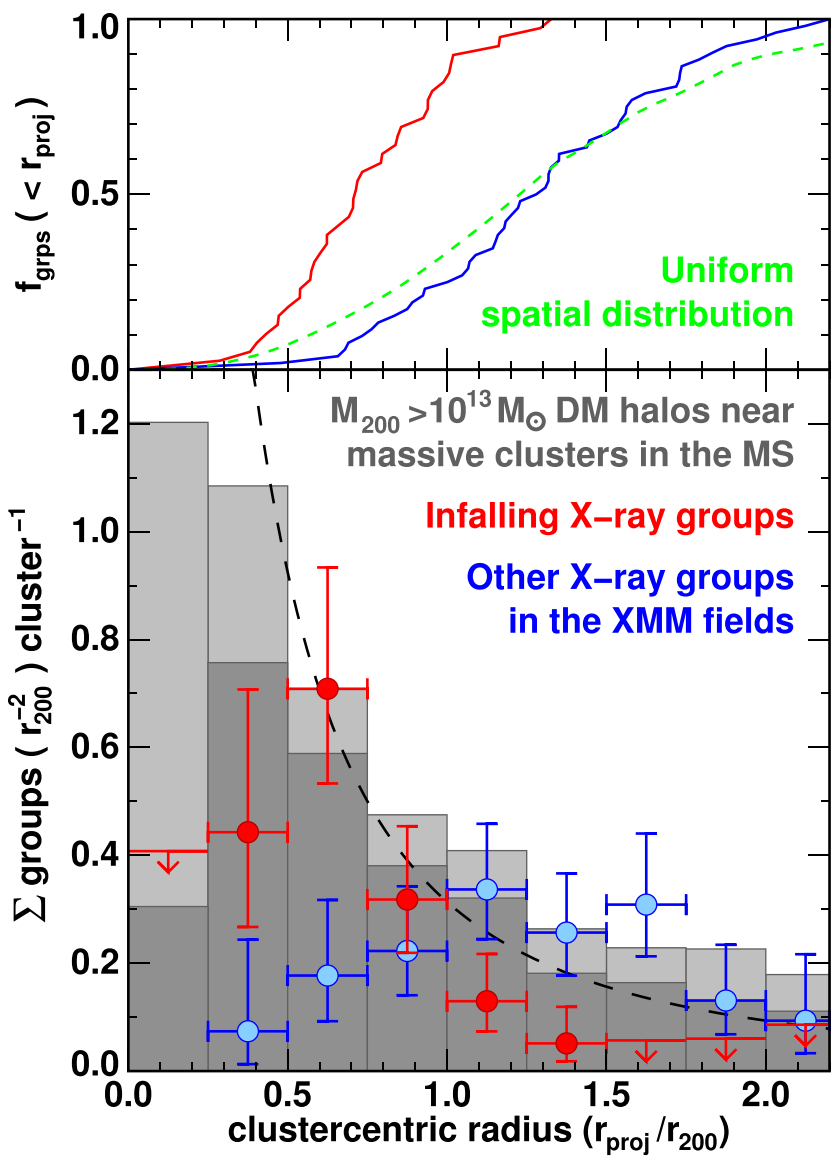

Figure 12. Composite surface number density distribution $\Sigma(r)$ of the 39 observed infalling X-ray groups (red points) and 52 other 'isolated' field $\mathrm{X}$-ray groups (blue points) as a function of projected cluster-centric radius (lower panel). For those radial bins containing no infalling groups, the Poisson $1 \sigma$ upper limit (Gehrels 1986) is shown. The light grey shaded histogram shows the predicted number density distribution of $M_{200}>10^{13} \mathrm{M}_{\odot}$ groups as a function of projected cluster-centric radius around the 75 most massive clusters in the MS at $z=0.21$. The dark shaded distribution indicates the predicted radial distribution of those groups that would be detected, applying the completeness correction of Fig. 10c. The dashed curve shows the bestfitting NFW profile to the radial distribution of cluster galaxies $\left(c_{\mathrm{g}}=3.01\right.$; Haines et al. 2015). The upper panel shows the cumulative radial distribution $f\left(<r_{\text {proj }}\right)$ of the infalling X-ray groups (red curve) and 'isolated' $\mathrm{X}$-ray groups detected in the same $X M M$ fields. The dashed green curve indicates the predicted cumulative radial distribution if groups were uniformly distributed across the XMM images, taking into account the effects of incompleteness in the cluster cores (Fig. 10c).

observational biases, and the difficulty in detecting X-ray groups within $0.5 r_{200}$ above the much greater emission from the cluster ICM pushes the expected peak out to $0.25-0.75 r_{200}$ (darker histogram). The sharp increase in the projected number density of groups moving towards the cluster centre parallels that seen also for the member galaxies (Haines et al. 2015, fig. 5), which was best fit by a Navarro-Frenk-White (NFW) profile with $c_{\mathrm{g}}=3.01 \pm 0.16$ (dashed curve; Haines et al. 2015). Such an NFW profile also describes well the predicted radial distribution of groups (grey histograms) over $0.5-2.2 r_{200}$, but is marginally inconsisent with our lack of infalling X-ray groups beyond $1.5 r_{200}$. This largely reflects the fact that clusters lie at the centres of large-scale $(\gtrsim 10 \mathrm{Mpc})$ overdensities that extend well beyond the virial radius (Frenk et al. 1999). These large-scale overdensities are collapsing inwards to-

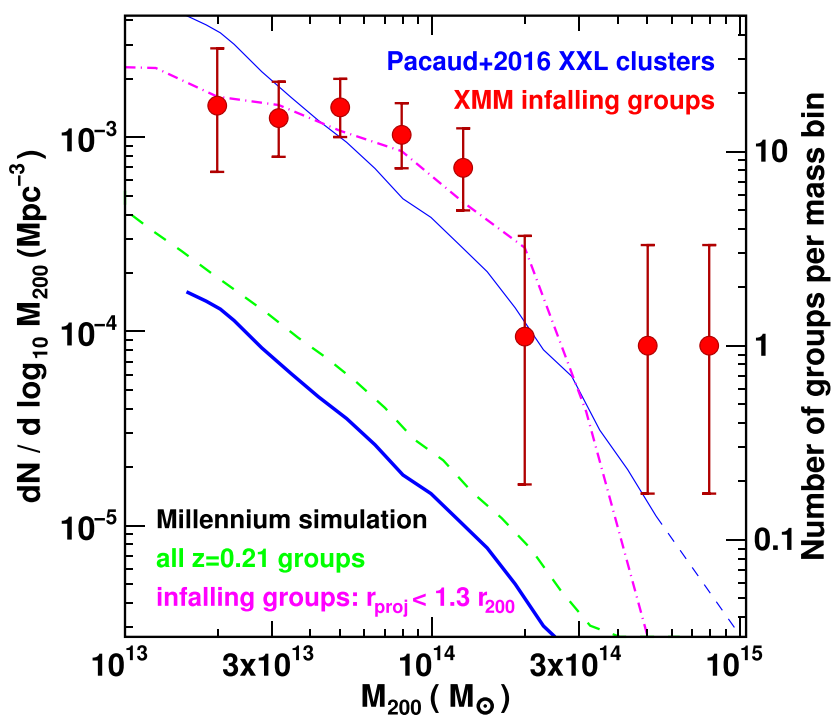

Figure 13. MF of the $39 X M M$-detected groups with redshifts associating them with the 23 primary clusters (red solid symbols). Error bars indicate Poisson uncertainties based on Gehrels (1986). The thick blue curve indicates the MF derived from the XLF of groups and clusters from the XXL survey (Pacaud et al. 2016). The thin blue curve shows the same MF, normalized upwards by a factor of 26 (see text). The dot-dashed magenta curve shows the MF of simulated infalling galaxy groups within $1.3 r_{200}$ (projected) of the 75 most massive clusters in the MS at $z=0.21$, while the dashed green curve indicates the $z=0.21$ halo MF averaged over the whole MS.

wards the cluster, dragging the infalling $\mathrm{X}$-ray groups and galaxies with them (Haines et al. 2015, figs 9 and 10).

\subsection{The mass function of infalling $\mathrm{X}$-ray groups}

Fig. 13 shows the MF of the infalling X-ray groups (red points), after correcting for incompleteness (Section 2.6). The left-hand axis shows the MF in units of groups per dex in mass per comoving $\mathrm{Mpc}^{3}$. The comoving volume containing the infalling group sample for each cluster is estimated as that within the redshift limits corresponding to the cluster caustics and extending over a circular area of sky of radius $1.3 r_{200}$. Summing these 23 volumes gives a grand total comoving volume of $5.9 \times 10^{4} \mathrm{Mpc}^{3}$.

The slope of the MF appears to flatten off for masses below $\sim 10^{14} \mathrm{M}_{\odot}$. The shape and overall normalization of the MF (in terms of groups per mass bin per cluster; right-hand axis) is consistent with that predicted by the MF of infalling galaxy groups with projected separations $<1.3 r_{200}$ from the 75 most massive clusters in the MS (dot-dashed magenta curve). The DM halo MF depends strongly on $\sigma_{8}$, and the value used in the MS (0.90) is inconsistent with current observational estimates $(\sim 0.81 \pm 0.01$; Planck Collaboration XIII 2016). We use abundance matching to first adjust the masses to a cosmological model with $\sigma_{8}=0.8$ using HMFCALC (Murray, Power $\&$ Robotham 2013). This acts to reduce the masses by $\sim 0.1 \mathrm{dex}$.

For comparison, the thick blue curve shows an estimate of the overall MF of clusters in the Universe at $z \sim 0.2$, obtained by applying the same $M_{200}-L_{X}$ relation to the X-ray luminosity function (XLF) of Pacaud et al. (2016). This XLF is based on a flux-limited sample of the 100 brightest extended X-ray sources found in the XXL survey (Pierre et al. 2016). This is the largest programme carried out by XMM-Newton, covering a total area of $50 \mathrm{deg}^{2}(412$ $X M M$ pointings) over two fields, to comparable depths $(10 \mathrm{ksec})$ 
as those used here, with the objective of providing a statistical and representative sample of groups and clusters out to $z \sim 0.5$ (and above), suitable for constraining cosmological parameters. Most of these systems are located between $z=0.1$ and 0.5 . The X-ray luminosities of Pacaud et al. (2016) were measured over the 0.5$2.0 \mathrm{keV}$ spectral band, and so were first divided by a global factor of 0.59 to $k$-correct them to the $0.1-2.4 \mathrm{keV}$ band used here and in Leauthaud et al. (2010). The most notable difference between the 'cosmic' MF and that of our infalling X-ray groups is the overall normalization. The comoving number density of X-ray groups in the infall regions of clusters is more than an order of magnitude higher than that seen in the XXL survey volume. The 'cosmic' MF has to be normalized upwards by a factor of $\sim 26$ (thin blue curve) in order to predict the same overall number of $M_{200}>10^{13.2} \mathrm{M}_{\odot}$ groups as that observed in the XMM infalling group sample.

Moreover, over the remainder of the $0.15 \leq z<0.30$ volume covered by our $23 X M M$ images, we detect only 10 more X-ray groups above the $3 \sigma$ SNR threshold, despite this volume being $5.76 \times$ larger than that confined within the redshift limits of the clusters. This corresponds to an overabundance of X-ray groups in the cluster infall regions of a factor $\sim 22$, comparable to the previous estimate, and confirming that the infall regions of clusters are $\sim 25 \times$ overdense in group-mass systems with respect to the cosmic average at that redshift.

The shapes of the two MFs also appear different. The flattening seen in the MF of the infalling X-ray groups is in marked contrast to the much steeper MF of XXL systems, which can be well described as a single power law $\left(N(M) \propto M^{-1.6}\right)$ without any sign of a break. This steep, power-law form closely resembles the global MF of DM haloes averaged over the full volume of the MS at $z=0.21$ (dashed green curve), where no break in the MF is apparent. The clear difference in the MFs of the X-ray groups around massive clusters presented here and of X-ray groups sampled over a large, representative volume of the Universe through the XXL survey reproduces well the predicted effect of the overdense cluster environment on the MF of DM haloes seen within the MS.

The apparent differences in the shape of the MF of X-ray groups according to large-scale environment is further elucidated in Fig. 14, which shows the cumulative mass fractions of $\mathrm{X}$-ray groups from the XXL survey (blue curve) and the 39 infalling X-ray groups (red line), as well as the corresponding DM halo populations from the MS. Each curve presents the fraction of $M_{200}>10^{13.2} \mathrm{M}_{\odot}$ groups (DM haloes) that are also above a given $M_{200}$ mass, as a function of $M_{200}$. This confirms that the MF of the X-ray groups found in the vicinity of massive clusters is systematically top-heavy with respect to that of the general population of X-ray groups at these redshifts from the XXL survey. A Kolmogorov-Smirnov test finds that the probability that both MFs is drawn from the same distribution to be just $0.0006\left(D_{\mathrm{KS}}=0.323\right.$; maximal distance between curves is shown by the vertical black line). This corresponds to a $3.5 \sigma$ result.

The systematic bias towards a top-heavy MF observed for $\mathrm{X}$-ray groups around massive clusters replicates that seen in the MS (Faltenbacher et al. 2010; Haines et al. 2015). The cumulative MF of DM haloes in the vicinity $\left(r_{\text {proj }}<1.3 r_{200}\right)$ of a massive cluster at $z=0.21$ (magenta dot-dashed curve) is found to be consistent with our observed MF for infalling X-ray groups, and top-heavy with respect to the MF of DM haloes averaged across the full MS at the same snapshot (green dashed curve). The large-scale overdensity centred on the massive cluster biases the halo MF in its vicinity, increasing the relative contribution of higher mass haloes at the expense of lower mass systems. This biasing has the effect of increasing the importance of accreting $\sim 10^{14} \mathrm{M}_{\odot}$ systems to

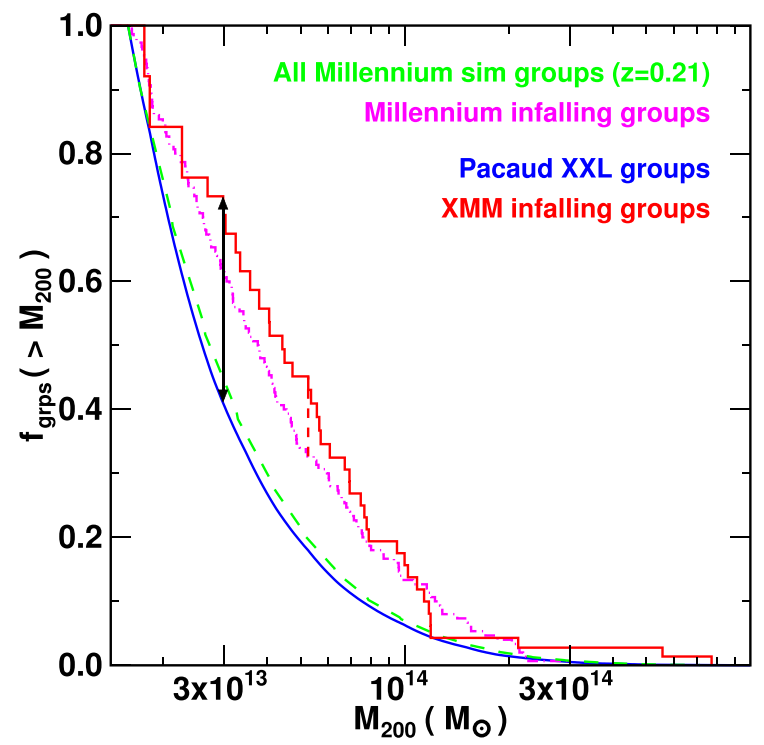

Figure 14. Cumulative mass distribution $f(>M)$ of the $39 X M M$-detected galaxy groups (red line), X-ray groups from the XXL survey (Pacaud et al. 2016, blue curve), infalling galaxy groups around 75 massive clusters in the MS (dot-dashed magenta line), and all $z=0.21$ DM haloes with $M_{200} \geq$ $10^{13.2} \mathrm{M}_{\odot}$ in the MS (green curve). The vertical dashed line indicates the maximal differences between the observed cumulative mass distributions.

the mass growth of rich clusters with respect to simple predictions based on the cosmic halo MF.

\subsection{The total mass contained within infalling groups}

The total mass of the 39 infalling groups detected by $X M M$ is $3.77 \times 10^{15} \mathrm{M}_{\odot}$, which after correcting for incompleteness comes to $5.13 \times 10^{15} \mathrm{M}_{\odot}$ in systems above $10^{13.2} \mathrm{M}_{\odot}$, or $2.23 \times 10^{14} \mathrm{M}_{\odot}$ for each of the 23 clusters in our sample. This corresponds to $19.5 \pm 5.1$ percent of the mean mass of the 23 primary clusters $\left(\left\langle M_{200}\right\rangle=11.47 \times 10^{14} \mathrm{M}_{\odot}\right)$, where the uncertainty is estimated by bootstrap resampling to account for the significant cluster-to-cluster scatter.

The two most massive X-ray 'groups' in Fig. 13 are Abell 1758S and Abell 115S, both of which are the lesser component of well-known double clusters undergoing major mergers (David \& Kempner 2004; Gutierrez \& Krawczynski 2005; Okabe \& Umetsu 2008). Although nominally clusters, we include these among our infalling X-ray 'groups' as they will be accreted and subsumed by the primary cluster $(\mathrm{A} 1758 \mathrm{~N}, \mathrm{~A} 115 \mathrm{~N})$ in the same way. Even so, these two clusters only contribute $1.33 \times 10^{15} \mathrm{M}_{\odot}$ between them, representing 26 per cent of the total mass within our infalling X-ray group sample. Excluding them does not dramatically change our estimate of the amount of mass being accreted on to clusters in the form of groups.

\subsection{The group-cluster mass ratio distribution}

Fig. 15 shows the distribution of $M_{200}$ mass ratios between the infalling galaxy groups and the primary clusters that they are associated with $\left(M_{\mathrm{gr}} / M_{\mathrm{cl}}\right.$; red points), using the $X M M$-based $M_{200, X}$ cluster masses from Table 1 . The mass-ratio distribution appears approximately consistent with a power law $\mathrm{d} N / \mathrm{d} \ln \left(M_{\mathrm{gr}} / M_{\mathrm{cl}}\right) \propto$ $\left(M_{\mathrm{gr}} / M_{\mathrm{cl}}\right)^{-\alpha}$ over the range $0.02-1.0$ in group-cluster mass ratio, with $\alpha=1.17_{-0.34}^{+0.28}$ (red dot-dashed line). This is in excellent 


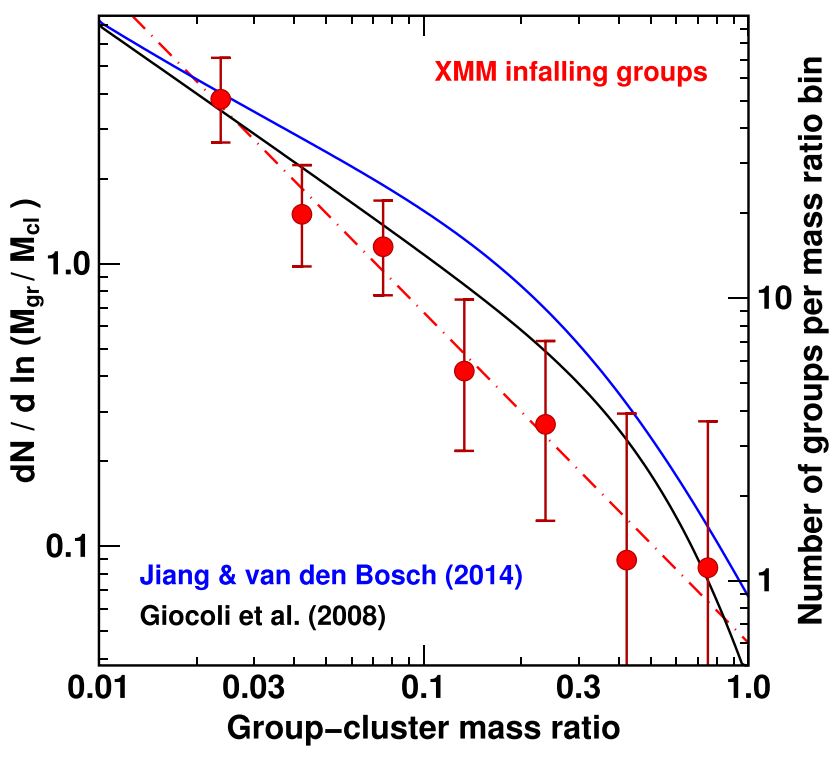

Figure 15. The distribution of the mass ratios between $X M M$-detected galaxy groups and the primary clusters, after correcting for incompleteness (red solid symbols). The best-fitting power law is shown by the red dot-dashed line. The black and blue curves show the predicted unevolved subhalo MFs of Giocoli et al. (2008) and Jiang \& van den Bosch (2014), respectively.

agreement with the best-fitting power-law index of $1.09_{-0.32}^{+0.42}$ obtained by Okabe et al. (2014) for the sub-halo MF through a weak lensing analysis of sub-haloes in the Coma cluster, and indices $\sim 0.9-1.0$ predicted by analytical models (Taylor \& Babul 2005) and numerical simulations.

The black solid curve shows the unevolved subhalo MF of Giocoli et al. (2008):

$$
\frac{\mathrm{d} N}{\mathrm{~d} \ln \left(M_{\mathrm{gr}} / M_{\mathrm{cl}, 0}\right)}=N_{0} x^{-\alpha} \exp \left(-6.283 x^{3}\right), \quad x=\left|\frac{M_{\mathrm{gr}}}{\alpha M_{\mathrm{cl}, 0}}\right|,
$$

where $M_{\mathrm{gr}}$ is the mass of the progenitor group halo at the time of accretion, $M_{\mathrm{cl}, 0}$ is the present day mass of the descendent cluster, $\alpha=0.8$ and $N_{0}=0.21$. Here, we assume that $M_{\mathrm{cl}, 0}=\left(M_{\mathrm{cl}}+M_{\mathrm{gp}}\right)$, i.e. the group's mass has been subsumed by the cluster by the present day. Jiang \& van den Bosch (2014) refined the fitting function of Giocoli et al. (2008), adding in an extra power-law term to better model the unevolved subhalo MF of haloes within the MS (blue curve).

The form and steepness of the mass-ratio distribution of infalling $X M M$ groups reproduce well the unevolved subhalo MFs of both Giocoli et al. (2008) and Jiang \& van den Bosch (2014), the main difference being a systematic shortfall at most mass ratios. This is unsurprising, as we are only detecting the groups that are being accreted into the clusters at late epochs, while the functions of Giocoli et al. (2008) and Jiang \& van den Bosch (2014) include the contributions of subhalos accreted at all redshifts, a significant fraction of which will have long been stripped of their X-ray emitting gas haloes.

\section{DISCUSSION}

A fundamental prediction of the $\Lambda \mathrm{CDM}$ model is that galaxy clusters, as the most massive collapsed haloes in the universe, form latest, doubling their mass since $z \sim 0.5$ (e.g. van den Bosch et al.
2014). As structure formation occurs hierarchically, much of this late mass growth must be achieved through the accretion of poorer clusters and group-mass systems, and so the outer regions of clusters must be replete with infalling group-mass systems. The key objective of our XMM survey of 23 massive clusters is to perform a simple empirical verification of the ongoing assembly of massive clusters through the accretion of groups, as predicted by $\Lambda \mathrm{CDM}$, and to estimate the contribution of these infalling groups to the mass growth rate of the primary clusters.

\subsection{The mass assembly history of clusters}

The average rates at which clusters assemble their mass through mergers and accretion as function of redshift have been investigated for a range of cosmologies using a combination of $N$-body simulations and Monte Carlo realizations based on the EPS framework.

McBride et al. (2009) investigated the mass accretion histories (MAHs) of DM haloes from the MS, finding that a two-parameter function of the form

$M(z)=M_{0}(1+z)^{\beta} \exp (-\gamma z)$

was versatile enough to accurately capture the main features of most MAHs in the simulation. They were also able to obtain a good fit to the mean mass growth rates of haloes as a function of halo mass and redshift, by differentiating the above equation. Correa et al. (2015) have shown using EPS theory and the redshift dependence of the linear growth factor $D(z)$ that the mass growth of haloes is well described by an exponential growth at high redshifts, while at low redshifts when dark energy dominates, the growth of density perturbations is slowed by the accelerated expansion of the Universe, necessitating an additional power-law term.

Fig. 16 shows the MAHs of the 75 most massive DM haloes in the MS (grey curves). The main branch of the merger tree of each cluster halo is determined by identifying the most massive progenitor of the descendent cluster halo in the previous snapshot. The most notable feature is the large cluster-to-cluster scatter among the MAHs, with some clusters assembling more than half of their mass in the last $2 \mathrm{Gyr}$, while others were largely in place by $z \sim 0.5$ and some are appearing to be losing mass at late epochs. These mass-loss events occur during major mergers, which produce first a dramatic increase in mass and a corresponding rapid increase in the velocity dispersion, followed by a slower phase of mass-loss as a significant amount of mass from the secondary halo rebounds out of the primary halo and orbits beyond its $r_{200}$ radius for 2-4 Gyr (Behroozi et al. 2015), analogously to 'back-splash' galaxies.

While the individual cluster MAHs show significant stochastic variation, the mean MAH (green curve) shows a steady monotonic increase in mass over the last $10 \mathrm{Gyr}$. The large scatter in individual MAHs implies the need to average over many clusters to derive useful constraints on the cosmic growth of mass within clusters, and the green shaded region indicates the $1 \sigma$ range in averaged MAHs produced by combining the growth rates of 23 clusters, selected at random from the full sample. The total $M_{200}$ masses of a random sample of $23 \mathrm{MS}$ clusters should have $77.6 \pm 5.9$ per cent of their present day $M_{200}$ mass at $z=0.223$, the mean redshift of our primary clusters (vertical dashed line). In other words, they should grow by a further $29 \pm 10$ per cent between $z=0.223$ and the present day. 




Figure 16. The MAHs of the 75 most massive DM haloes in the MS (grey curves). The solid green curve indicates the mean MAH of these 75 haloes, while the green shaded region indicates the expected $1 \sigma$ range of MAHs when averaging over 23 clusters selected at random from the full sample. The dashed blue, dot-dashed orange, and magenta curves indicate the mass growth for haloes of present day mass $M_{200}=10^{15} h^{-1} \mathrm{M}_{\odot}$ predicted by equation (4) (Fakhouri, Ma \& Boylan-Kolchin 2010), van den Bosch et al. (2014), and Zhao et al. (2009), respectively. The vertical black dashed line indicates the mean redshift $(\bar{z}=0.223)$ of the LoCuSS primary clusters and $X M M$ infalling groups.

Using the higher resolution Millennium II simulation, Fakhouri et al. (2010) obtained updated analytic fits to the mean mass growth rates of haloes of mass $M$ at redshift $z$ of:

$$
\begin{aligned}
\langle\dot{M}\rangle_{\text {mean }}= & 46.1 \mathrm{M}_{\odot} \mathrm{yr}^{-1}\left(\frac{M}{10^{12} \mathrm{M}_{\odot}}\right)^{1.1} \\
& \times(1+1.11 z) \sqrt{\Omega_{\mathrm{m}}(1+z)^{3}+\Omega_{\Lambda}} .
\end{aligned}
$$

The resulting MAH for a cluster halo of present day mass $M_{200}=10^{15} \mathrm{M}_{\odot}($ dashed blue curve $)$ is consistent with the mean MAH of the 75 most massive MS clusters. Zhao et al. (2009) derived a universal empirical model for the MAHs of DM haloes, by analysis of numerous $\mathrm{N}$-body simulations of a wide variety of cosmological models, which predicts a somewhat later mass assembly for $10^{15} h^{-1} \mathrm{M}_{\odot}$ haloes (dot-dashed magenta curve) in a Millennium cosmology. van den Bosch et al. (2014) used EPS merger trees calibrated with the Bolshoi $\mathrm{N}$-body simulation to derive average MAHs for haloes of a given mass in any $\Lambda$ CDM cosmology (dot-dashed orange curve).

These models all predict similar MAHs for $10^{15} h^{-1} \mathrm{M}_{\odot}$ clusters, whereby they have grown at a virtually constant rate since $z \sim 1$ (Fig. 16). The MAHs of van den Bosch et al. (2014), Fakhouri et al. (2010), and Zhao et al. (2009), respectively, predict mass increases of 27 per cent, 32 per cent, and 37 per cent between $z=0.223$ and the present day. These correspond to mass growth rates of 1317 per cent per Gyr for clusters at $z \sim 0.2$.

\subsubsection{Dependence on cosmological parameters}

The curves shown in Fig. 16 demonstrate well the expected clusterto-cluster scatter among their MAHs. Strictly speaking they are only valid for the exact cosmological model used in the simulation. The parameters were set in the MS $\left(\Omega_{\mathrm{m}}=0.25, \Omega_{\Lambda}=0.75, \sigma_{8}=0.90\right.$, $n_{s}=1.0, h=0.73$ ) to be close to those obtained from WMAP1 (Spergel et al. 2003), but are now somewhat divergent from the current best estimates obtained from analysis of the full-mission Planck CMB data $\left(\Omega_{\mathrm{m}}=0.308 \pm 0.012, \sigma_{8}=0.8149 \pm 0.0093\right.$, $n_{s}=0.9677 \pm 0.0060, H_{0}=67.81 \pm 0.92$; Planck Collaboration XIII 2016).

The universal models of Zhao et al. (2009) and the EPS-based models of van den Bosch et al. (2014) allow us to investigate the dependence of the average MAHs of cluster-mass haloes on the main cosmological parameters, and recalibrate the results on to the Planck 2015 cosmological model. Fig. 17 shows the effect of varying $\Omega_{\mathrm{m}}$ and $\sigma_{8}$ on the average MAHs of DM haloes with present day masses $M_{200}=10^{15} \mathrm{M}_{\odot}$ in flat $\Lambda \mathrm{CDM}$ cosmologies, while keeping the remaining parameters fixed to the Planck 2015 values, using the Zhao et al. (2009) code with an Eisenstein \& Hu (1998) power spectrum. These confirm that both $\Omega_{\mathrm{m}}$ and $\sigma_{8}$ have a significant effect on the MAHs of cluster-mass haloes, with the rate of growth at late epochs rising with increasing $\Omega_{\mathrm{m}}$ and decreasing $\sigma_{8}$. In contrast, varying the tilt of the primordial power-law spectrum $n_{s}$ has negligible influence on the MAHs of cluster-mass haloes.

Increasing $\Omega_{\mathrm{m}}$ from 0.25 (MS) to 0.308 (Planck 2015) results in a corresponding increase in the rate of growth of clusters from $z=0.223$ to the present day of 7.4 per cent, while decreasing $\sigma_{8}$ from 0.90 to 0.8149 increases the growth rate by an additional 10.9 per cent. The combined changes in moving from the MS cosmological model to that of Planck 2015 increases the late-time growth rate by 18.3 percent, resulting in mass growth rates for $z \sim 0.2$ clusters of 15-20 per cent per Gyr.

\subsection{The contribution of X-ray groups to the mass growth rate of clusters}

Our finding of $39 X M M$-detected galaxy groups in the infall regions of 23 massive clusters, corresponding to an average mass of $2.23 \times 10^{14} \mathrm{M}_{\odot}$ per cluster, or 19 per cent of the mean $M_{200}$ mass of the primary cluster, suggests that these galaxy groups can explain a significant fraction of the mass growth of galaxy clusters between $z \sim 0.2$ and the present day.

The comparison sample of group-mass haloes from the MS allow us examine the likely fates of the $39 X M M$-detected galaxy groups, by following the orbits from the $z=0.21$ snapshot where they are identified to the present day. We find that two-thirds ( 67 per cent) of the simulated groups located within the $X M M$ fields and recovered by the wavelet-reconstruction algorithm will be accreted into the cluster (pass within $r_{200}$ ) by the present day. In contrast, 22 per cent of the detected $X M M$ groups are expected to be physically more than $4 r_{200}$ from the cluster at the time of observation and, despite being on their first infall, remain outside $r_{200}$ at $z=0$. This is balanced by the 24 percent of those simulated infalling $M_{200}>10^{13} \mathrm{M}_{\odot}$ haloes accreted into the clusters between $z=0.21$ and 0 being outside the XMM field of view at $z=0.21$ and therefore missed from our sample. This can be understood given that those haloes only accreted into the clusters in the last snapshot $(z<0.02)$ were on average at $2.89 \pm 0.70 r_{200}$ at $z=0.21$.

Taking the above correction factors into account, we estimate that clusters increase their mass by $16.2 \pm 4.2$ percent between 

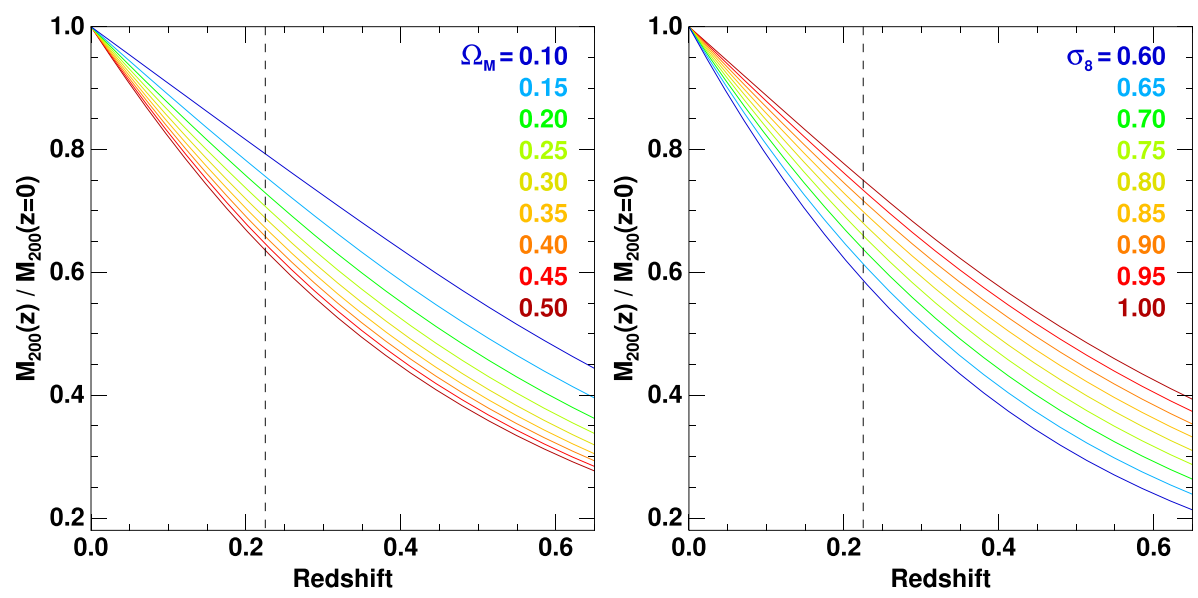

Figure 17. Average MAHs, $\left\langle M_{200}(z) / M_{200}(z=0)\right\rangle$, for haloes of final mass $M_{0}=10^{15} h^{-1} \mathrm{M}_{\odot}$ in different, flat $\Lambda$ CDM cosmologies, based on the empirical models of Zhao et al. (2009). The left-hand panel shows the effect of varying $\Omega_{\mathrm{m}}$ from 0.1 to 0.5 in steps of 0.05 , while the right-hand panel shows the effect of varying $\sigma_{8}$ from 0.6 to 1.0 in steps of 0.05 . All of the other cosmological parameters are kept fixed at the values from the Planck 2015 model. The vertical dashed line indicates the mean redshift $(\bar{z}=0.223)$ of our sample of 23 clusters.

$z=0.223$ and the present day (or 6 percent per Gyr) due to the accretion of groups more massive than $10^{13.2} \mathrm{M}_{\odot}$. This confirms that X-ray groups are contributing significantly to the mass growth rate of clusters. However, this estimate for the mass accreted in the form of groups is only half that predicted for the overall mass growth of massive clusters over the same period (32-44 per cent), as described in the previous section.

\subsection{Accounting for the rest of the mass accreted by clusters and estimating their growth rates}

Our empirical estimate that clusters are able to increase their masses by $\sim 16$ per cent between $z=0.223$ and the present day through the accretion of $M_{200}>10^{13.2} \mathrm{M}_{\odot}$ X-ray groups is not sufficient to fully explain the mass growth rate of clusters. Thus, either the growth rate of massive clusters is much lower than that predicted by cosmological simulations or there are other major contributions to the mass accretion rate of clusters from less massive DM haloes (e.g. those hosting individual galaxies) or matter that is not bound within any DM halo.

Within the EPS formulism (Press \& Schechter 1974; Bond et al. 1991; Bower 1991; Lacey \& Cole 1993), all of the growth of DM haloes comes from mergers by construction. However, using merger trees constructed from both Millennium simulations, and taking care to accurately account for halo fragmentation, Genel et al. (2010) find that all resolved mergers, down to mass ratios of $10^{-5}$ between them, contribute only $\approx 60$ per cent of total halo mass growth, regardless of halo mass and redshift. Major mergers with ratios above 1:3 (1:10) contribute just 20 per cent ( 30 per cent). Instead, they indicate that 40 per cent of the mass in haloes (up to and including cluster-mass haloes) comes from genuinely smooth accretion of DM that was never bound in smaller haloes. While there is some freedom of how merger trees are constructed, Genel et al. (2010) verified this result by following the individual DM particles within two cosmological simulations and labelling each one that had belonged to an identified bound structure at any point in its past, prior to its accretion into the primary halo.

Our estimate that the accretion of $M_{200}>10^{13.2} \mathrm{M}_{\odot}$ X-ray groups is sufficient to account for roughly $35-50$ per cent of the predicted mass growth rate of $\sim 10^{15} \mathrm{M}_{\odot}$ clusters appears consistent with the findings of Genel et al. (2010). This assumes that our $L_{X}$-based group mass estimates are unbiased relative to the true masses. Given their proximity to the primary cluster, some of these groups could be affected by ram-pressure stripping that progressively removes the X-ray emitting gas (see Section 4.6). As a sanity check of our mass estimates, we split the groups into three mass bins, and for those groups with four or more confirmed members, measure the distribution of the LOS velocity offsets relative to the group's mean redshift. The resultant velocity dispersions are $257 \mathrm{~km} \mathrm{~s}^{-1}$ for the 12 groups with $\log M_{200}<13.75,328 \mathrm{~km} \mathrm{~s}^{-1}$ for the 14 groups with $13.75 \leq \log M_{200}<14.05$ and $369 \mathrm{~km} \mathrm{~s}^{-1}$ for the 7 groups with $\log M_{200}>14.05$. These values are consistent with the $M_{200}-\sigma$ trend of SDSS groups (Yang et al. 2007) and the $L_{X}-\sigma$ relations of Zhang et al. (2011) and Clerc et al. (2016). The LOS velocity distributions of each stacked group sample are consistent with being a Gaussian function. Our sample may however miss the mass contribution from groups that have been recently accreted but are now orbiting back out beyond $r_{200}$. Their DM halo may still be largely intact, but the $\mathrm{X}$-ray emitting gas has been sufficiently stripped as to be undetected. The LOS velocity offsets of such groups are likely to be rather low as they approach apocentre.

De Boni et al. (2016) have suggested that it is possible to estimate the overall mass accretion rate of clusters from their mass profiles beyond the virial radius. The aggregate radial velocity of DM within a radial shell reaches a minimum at $2-3 r_{200}$, that is beyond the splashback radius, and so most closely represents the infall of new material on to the cluster. By measuring the mass profile of the cluster over 2-3 $r_{200}$ using the caustic method of Diaferio \& Geller (1997), and assuming the infall velocity based on spherical collapse model, they are able to approximately reproduce the mass accretion rates of clusters within simulations. Given the current availability of dense redshift surveys of clusters galaxies beyond $2 r_{200}$ for many rich clusters (e.g. Rines et al. 2013), estimates of their typical mass accretion rates should be feasible.

\subsection{The MF of infalling X-ray groups}

The variation of the DM halo MF with large-scale density such that the MF appears top-heavy in overdense regions can be readily understood from a theoretical perspective. Collapsed DM haloes are 
biased tracers of mass. This implies that the abundance of DM haloes in overdense and underdense regions is not expected to simply differ by a factor that reflects the change in large-scale matter density. Instead,

$n(M \mid \delta) \approx[1+b(M, z) \delta] n(M)$,

where $n(M \mid \delta)$ is the abundance of DM haloes of mass $M$ in a region of overdensity $\delta, n(M)$ is the cosmically averaged abundance, and $b(M, z)$ is the mass-dependent bias parameter of haloes at redshift $z$ (Mo \& White 1996; Sheth \& Tormen 1999; Abbas \& Sheth $2005)$. As the bias $b(M)$ typically increases monotonically with mass (Tinker et al. 2010), this acts to increase the ratio of high-mass DM haloes to low-mass haloes in overdense regions, relative to less dense regions. Thus, the MF in overdense regions should be topheavy. The effects of large-scale density on the halo MF were examined by Faltenbacher et al. (2010) using the MS, confirming that the fraction of matter within group-mass haloes $\left(M_{200} \gtrsim 10^{13.5} \mathrm{M}_{\odot}\right)$ increases significantly with large-scale density, and the halo MF becomes increasingly top heavy (see also Lemson \& Kauffmann 1999).

Chon, Böhringer \& Nowak (2013) found that clusters within superclusters were systematically more X-ray luminous than clusters outside superclusters. Assuming that this overabundance of X-ray luminous clusters represents an excess of massive clusters within superclusters provides observational support for the theoretical expectation that the MF of clusters in overdense regions (superclusters) is top heavy. Similarly, this mass bias was also observed for galaxy groups in the vicinity of clusters in the $2 \mathrm{dF}$ Galaxy Redshift Survey (Ragone et al. 2004).

\subsection{Impact for galaxy evolution}

These X-ray groups are not only contributing a large proportion of the the DM required for cluster mass growth but also host a significant fraction of the galaxies that arrive on to the clusters at late epochs. McGee et al. (2009) estimate that $\sim 50$ per cent of cluster galaxies accreted since $z=0.5$ arrived on to the cluster as member of an infalling group with $M_{200}>10^{13} \mathrm{M}_{\odot}$. Galaxy groups have been shown to have a major impact on the evolution of their member galaxies, suppressing star formation activity through the interaction of the galaxy with the intra-group medium (ram-pressure stripping or starvation), or transforming their morphologies through lowvelocity encounters and mergers with other group members. The fraction of star-forming galaxies among group members is lower than that seen in the field (at fixed stellar mass and redshift; Haines et al. 2007; Ziparo et al. 2014), and declines with increasing group mass and proximity to the group centre (Weinmann et al. 2006; Woo et al. 2013).

Thus, many galaxies are arriving on to clusters having already been transformed from star-forming spirals into passive early-types within groups, a mechanism known as pre-processing (Zabludoff et al. 1996, 1998; Dressler et al. 2013; Just et al. 2015; Jaffé et al. 2016). This can contribute significantly to the cluster population of passive early-types, but also explains the short-fall of star-forming galaxies at large cluster-centric radii $\left(\gtrsim 2-3 r_{200}\right)$, where no galaxies should have previously encountered the cluster (Chung et al. 2011; Haines et al. 2015). This should be exacerbated by the top-heavy MF of these infalling groups, meaning that galaxies are more likely to be in massive $\mathrm{X}$-ray luminous groups than the cosmic average. We will examine the impact of pre-processing on the galaxies within these infalling X-ray groups in Bianconi et al. (2018).

\subsection{The next steps}

This work presents a first attempt to quantify the numbers and demographics of X-ray groups in the immediate vicinity of a statistical sample of massive clusters, and derives empirical constraints on the rates at which clusters are growing through the accretion of groupmass systems. By identifying groups through their extended X-ray emission, we can confidently associate them to massive virialized DM haloes. As we only have the X-ray luminosities of these groups, we have had to make certain simplifying assumptions to estimate their masses. In particular, by using the $M_{200}-L_{X}$ scaling relation of Leauthaud et al. (2010), we are assuming that the X-ray emitting gas content of these infalling groups remains bound within the host DM halo, and maintains the same density and temperature structures as isolated field groups (such as those from the COSMOS survey). At the same time, we expect that as these groups are accreted into the cluster, passing through the increasingly dense ICM, their $\mathrm{X}$-ray emitting hot gas haloes are progressively ram-pressure stripped (Gunn \& Gott 1972; Poole et al. 2006; McCarthy et al. 2008). The plasma physics of this process is however very complex. Magnetic fields, turbulence, viscosity, KH instabilities, and conduction are all likely to play a role in determining when and how rapidly $\mathrm{X}$-ray emitting gas is stripped from the group, the appearance of the extended tail of high-density stripped gas and how long this wake can survive before mixing with the ambient ICM (Roediger et al. 2015a,b).

Examples of this gas stripping have been seen in recent X-ray observations of groups infalling into Abell 85, Abell 2142, Abell 4067, ZwCl 8338, and Abell 780 (Schellenberger \& Reiprich 2005; Eckert et al. 2014; Chon \& Böhringer 2015; Ichinohe et al. 2015; De Grandi et al. 2016). These group-cluster mergers also leave shock fronts, spiral features indicative of gas sloshing, and increased gas clumping in the cluster outskirts (Reiprich et al. 2013). On the other hand, during the group-cluster mergers, the X-ray luminosity may be briefly boosted as the group makes its pericentre passage (Ricker $\&$ Sarazin 2001), biasing the resulting group MF (Randall, Sarazin $\&$ Ricker 2002). The hydrodynamical simulations of group-cluster mergers by Poole et al. (2006) show how as a group approaches pericentre, the gas on its leading edge is heated and compressed, temporarily boosting its X-ray luminosity, before being steadily stripped, leaving an extended trail of cool, low-entropy gas behind it, similar to those seen by Eckert et al. (2014) and De Grandi et al. (2016). In particular, Eckert et al. (2014) estimate that $>90$ per cent of the gas mass from the group falling into Abell 2142 has been stripped to form an $800 \mathrm{kpc}$ long tail. These detailed observations of individal group-cluster mergers are providing fundamental insights and constraints on the ICM plasma physics involved, revealing that the thermalization and mixing of the stripped group gas must be slow and inefficient (Eckert et al. 2017), and should lead to a better understanding of when and how the X-ray emitting gas is stripped from infalling groups.

The centres of an isolated group's galaxy population, X-ray gas, and DM halo should be coincident. As a group falls into a cluster, the ram pressure acts as a drag on the X-ray gas, causing it to lag behind the member galaxies and the DM, both of which are effectively collisionless. For the 32 infalling X-ray groups for which a clear BGG could be identified, there is a median separation of $65 \mathrm{kpc}$ between the BCG and X-ray centroid, with 68 per cent of the separations in the range $28-143 \mathrm{kpc}$. These separations are consistent with those seen for infalling groups in the BAHAMAS simulation (McCarthy et al. 2017). HST observations of these groups would allow their DM distributions to be constrained, to both confirm the 
overall masses of these groups and evidence of this lag in the hot gas component relative to the DM. Such observations can also test the self-interaction cross-section of DM, which would create a drag on the DM within the infalling groups, displacing the DM distribution relative to the group galaxies (Harvey et al. 2014, 2015).

This $X M M$ survey of infalling groups should also be a precursor to the much larger samples that should be obtained in the near future with eROSITA (Merloni et al. 2012). eROSITA will aim to perform a deep X-ray survey of the entire sky, with a combination of resolution and sensitivity good for studying the galaxy groups around $z<0.1$ clusters. It will lead to large statistical improvements primarily on lower mass clusters, compared to the current sample, as the local volume is small. Also, in a combination with spectroscopic follow-up on 4MOST/VISTA, it will deliver better statistics on larger separations from the cluster centre.

\section{SUMMARY}

We present an $X M M-N e w t o n$ survey to search for X-ray groups in the infall regions of 23 massive galaxy clusters at $0.15 \leq z<0.30$ from the LoCuSS cluster survey. All these clusters have excellent ancillary data including extensive spectroscopic coverage of cluster galaxies through ACReS and deep wide-field optical imaging from Subaru/Suprime-Cam, enabling us to identify the member galaxies associated with the X-ray emission of each group, and determine its redshift.

We identify $39 \mathrm{X}$-ray groups across the $23 X M M$ fields that have been spectroscopically confirmed to lie at the cluster redshift (and hence are likely falling into the primary cluster), above an SNR limit of 3. These groups all have at least one spectroscopic member, and a median of nine members. These infalling groups have $M_{200}$ masses in the range $2 \times 10^{13}-7 \times 10^{14} \mathrm{M}_{\odot}$, based on estimates derived from their X-ray luminosities. The key results from a statistical analysis of these groups are as follows:

(i) The 39 infalling X-ray groups lie at $0.3-1.3 r_{200}$ and are much more concentrated towards the cluster than the other 52 groups in the same fields (at the $6 \sigma$ level). The distribution of the LOS velocity offsets of the infalling groups relative to the primary clusters is non-Gaussian, consistent with them being an infalling population.

(ii) The comoving number density of the infalling X-ray groups is $\sim 25 \times$ higher than that seen in field regions.

(iii) The MF of the infalling X-ray groups is top-heavy with respect to that seen for isolated groups in the XXL survey at the $3.5 \sigma$ level. This is consistent with expectations of collapsed DM haloes being biased tracers of the underlying large-scale density field.

(iv) The average mass per cluster contained within these infalling $\mathrm{X}$-ray groups is $2.2 \times 10^{14} \mathrm{M}_{\odot}$, or $19 \pm 5$ per cent of the mass of the primary cluster.

(v) We estimate that $\sim 10^{15} \mathrm{M} \odot$ clusters increase their masses by $16 \pm 4$ percent between $z=0.223$ and the present day due to the accretion of X-ray groups with $M_{200} \gtrsim 10^{13.2} \mathrm{M}_{\odot}$. This represents 35-50 per cent of the expected mass growth of these clusters at these late epochs. The rest of the mass growth is likely to occur through the accretion of lower mass haloes and DM not bound within DM haloes.

This work represents the first attempt to statistically establish the frequency and demographics of X-ray groups in the infall regions of a representative sample of massive clusters, estimate their contribution to the mass growth of clusters and their mass function. It complements ongoing detailed X-ray studies examining the astrophysical processes acting on group-mass systems as they are accreted into massive clusters (e.g. Eckert et al. 2014; De Grandi et al. 2016).

\section{ACKNOWLEDGEMENTS}

CPH acknowledges financial support from PRIN INAF 2014 and CONICYT Anillo project ACT-1122. CPH acknowledges a travel grant through the FINCA 2017 visitor programme and the hospitality of Helsinki University where this work was completed. GPS, $\mathrm{MB}$, and FZ acknowledge support from the Science and Technology Facilities Council grant number ST/N000633/1.

\section{REFERENCES}

Abbas U., Sheth R. K., 2005, MNRAS, 364, 1327

Allen S. W., Evrard A. E., Mantz A. B., 2011, ARA\&A, 49, 409

Allevato V. et al., 2012, ApJ, 758, 47

Ascasibar Y., Diego J. M., 2008, MNRAS, 383, 369

Beers T. C., Flynn K., Gebhardt K., 1990, AJ, 100, 32

Behroozi P. S. et al., 2015, MNRAS, 454, 3020

Bianconi M., Smith G. P., Haines C. P., McGee S. L., Finoguenov A., Egami E., 2018, MNRAS, 473, L79

Böhringer H. et al., 2004, A\&A, 425, 367

Bonamente M., Landry D., Maughan B., Giles P., Joy M., Nevalainen J., 2013, MNRAS, 428, 2812

Bond J. R., Cole S., Efstathiou G., Kaiser N., 1991, ApJ, 379, 440

Bower R. G., 1991, MNRAS, 248, 332

Boylan-Kolchin M., Springel V., White S. D. M., Jenkins A., Lemson G., 2009, MNRAS, 398, 1150

Chon G., Böhringer H., 2015, A\&A, 574, 132

Chon G., Böhringer H., Smith G. P., 2012, A\&A, 548, 59

Chon G., Böhringer H., Nowak N., 2013, MNRAS, 429, 3272

Chung S. M., Eisenhardt P. R., Gonzalez A. H., Stanford S. A., Brodwin M., Stern D., Jarrett T., 2011, ApJ, 743, 34

Clerc N. et al., 2016, MNRAS, 463, 4490

Correa C. A., Wyithe S. B., Schaye J., Duffy A. R., 2015, MNRAS, 450, 1514

Czoske O., 2004, in Diaferio A., ed., IAU Colloq. 194, Outskirts of Galaxy Clusters: Intense Life in the Suburbs, Cambridge Univ. Press, Cambridge, p. 183

David L. P., Kempner J., 2004, ApJ, 613, 831

De Boni C., Serra A., Diaferio A., Giocoli C., Baldi M., 2016, ApJ, 818, 188

De Grandi S. et al., 2016, A\&A, 592, 154

Diafero A., Geller M. J., 1997, ApJ, 481, 633

Dressler A., Oemler A., Jr, Poggianti B., Gladders M. D., Abramson L., Vulcani B., 2013, ApJ, 770, 62

Ebeling H., Edge A. C., Bohringer H., Allen S. W., Crawford C. S., Fabian A. C., Voges W., Huchra J. P., 1998, MNRAS, 301, 881

Ebeling H., Edge A. C., Allen S. W., Crawford C. S., Fabian A. C., Huchra J. P., 2000, MNRAS, 318, 333

Eckert D. et al., 2014, A\&A, 570, 119

Eckert D. et al., 2017, A\&A, 605, 25

Edwards L. O. V., Fadda D., Frayer D. T., Lima Neto G. B., Durret F., 2010, ApJ, 140, 1891

Eisenstein D. J., Hu W., 1998, ApJ, 496, 605

Evrard A. E., Arnault P., Huterer D., Farahi A., 2014, MNRAS, 441, 3562

Fadda D., Biviano A., Marleau F. R., Storrie-Lombardi L. J., Durret F., 2008, ApJ, 672, L9

Fakhouri O., Ma C.-P., Boylan-Kolchin M., 2010, MNRAS, 406, 2267

Faltenbacher A., Finoguenov A., Drory N., 2010, ApJ, 712, 484

Finoguenov A. et al., 2007, ApJS, 172, 182

Finoguenov A. et al., 2009, ApJ, 704, 564

Finoguenov A. et al., 2010, MNRAS, 403, 2063

Finoguenov A. et al., 2015, A\&A, 576, 130 
Forman Q., Bechtold J., Blair W., Giacconi R., van Speybroeck L., Jones C., 1981, ApJ, 243, L133

Frenk C. S. et al., 1999, ApJ, 525, 554

Gao L., Navarro J. F., Frenk C. S., Jenkins A., Springel V., White S. D. M., 2012, MNRAS, 425, 2169

Gehrels N., 1986, ApJ, 303, 336

Genel S., Bouché N., Naab T., Sternberg A., Genzel R., 2010, ApJ, 719, 229

Giles P. A., Maughan B. J., Birkinshaw M., Worrall D. M., Lancaster K., 2012, MNRAS, 419, 503

Giocoli C., Tormen G., van den Bosch F. C., 2008, MNRAS, 386, 2135

Governato F., Babul A., Quinn T., Tozzi P., Baugh C. M., Katz N., Lake G., 1999, MNRAS, 307, 949

Gunn J. E., Gott J. R., 1972, ApJ, 176, 1

Gutierrez K., Krawczynski H., 2005, ApJ, 619, 161

Haines C. P., Gargiulo A., La Barbera F., Mercurio A., Merluzzi P., Busarello G., 2007, MNRAS, 381, 7

Haines C. P. et al., 2009a, ApJ, 704, 126

Haines C. P., Smith G. P., Egami E., Okabe N., Takada M., Ellis R. S., Moran S. M., Umetsu K., 2009b, MNRAS, 396, 1297

Haines C. P. et al., 2010, A\&A, 518, L19

Haines C. P. et al., 2012, ApJ, 754, 97

Haines C. P. et al., 2013, ApJ, 775, 126

Haines C. P. et al., 2015, ApJ, 806, 101

Harvey D. et al., 2014, MNRAS, 441, 404

Harvey D., Massey R., Kitching T., Taylor A., Tittley E., 2015, Science, 347,1462

Henry J. P., Finoguenov A., Briel U. G., 2004, ApJ, 615, 181

Henry J. P., Evrard A. E., Hoekstra H., Babul A., Mahdavi A., 2009, ApJ, 691,1307

Hikage C., Yamamoto K., 2016, MNRAS, 455, L77

Ichinohe Y., Werner N., Simionescu A., Allen S. W., Canning R. E. A., Ehlert S., Mernier F., Takahashi T., 2015, MNRAS, 448, 2971

Jaffé Y. L. et al., 2016, MNRAS, 461, 1202

Jiang F., van den Bosch F. C., 2014, MNRAS, 440, 193

Jones C., Forman W., 1999, ApJ, 511, 65

Just D. W. et al., 2015, preprint (arXiv:1506.02051)

Kettula K. et al., 2015, MNRAS, 451, 1460

Kravtsov A. V., Borgani S., 2012, ARA\&A, 50, 353

Lacey C. G., Cole S., 1993, MNRAS, 262, 627

Leauthaud A. et al., 2010, ApJ, 709, 97

Lemson G., Kauffmann G., 1999, MNRAS, 302, 111

Lemze D. et al., 2013, ApJ, 776, 91

Lovisari L., Reiprich T. H., Schellenberger G., 2015, A\&A, 573, 118

McBride J., Fakhouri O., Ma C.-P., 2009, MNRAS, 398, 1858

McCarthy I. G., Frenk C. S., Font A. S., Lacey C. G., Bower R. G., Mitchell N. L., Balogh M. L., Theuns T., 2008, MNRAS, 383, 593

McCarthy I. G., Schaye J., Bird S., Le Brun A. M. C., 2017, MNRAS, 465, 2936

McGee S. L., Balogh M. L., Bower R. G., Font A. S., McCarthy I. G., 2009, MNRAS, 400, 937

Mahdavi A., Hoekstra H., Babul A., Bildfell C., Jeltema T., Henry J. P., 2013, ApJ, 767, 116

Mann A. W., Ebeling H., 2012, MNRAS, 420, 2120

Mantz A., Allen S. W., Rapetti D., Ebeling H., 2010, MNRAS, 406, 1759

Martinet N. et al., 2016, A\&A, 590, 69

Martino R., Mazzotta P., Bourdin H., Smith G. P., Bartalucci I., Marrone D. P., Finoguenov A., Okabe N., 2014, MNRAS, 443, 2342

Maughan B. J., 2007, ApJ, 668, 772

Merloni A. et al., 2012, preprint (arXiv:1209.3114)

Mo H. J., White S. D. M., 1996, MNRAS, 282, 347

Murray S. G., Power C., Robotham A. S. G., 2013, Astron. Comput., 3, 23

O'Sullivan E. et al., 2017, MNRAS, 472, 1482

Okabe N., Smith G. P., 2016, MNRAS, 461, 3794

Okabe N., Umetsu K., 2008, PASJ, 60, 345

Okabe N., Takada M., Umetsu K., Futamase T., Smith G. P., 2010, PASJ, 62,811

Okabe N., Smith G. P., Umetsu K., Takada M., Futamase T., 2013, ApJ, 769, L35
Okabe N., Futamase T., Kajisawa M., Kuroshima R., 2014, ApJ, 784, 90

Pacaud F. et al., 2016, A\&A, 592, 2

Pearson R. et al., 2017, MNRAS, 469, 3489

Pedersen K., Dahle H., 2007, ApJ, 667, 26

Pereira M. J. et al., 2010, A\&A, 518, L40

Pierre M. et al., 2016, A\&A, 592, 1

Planck Collaboration XX, 2014a, A\&A, 571, 20

Planck Collaboration XVI, 2014b, A\&A, 571, 16

Planck Collaboration XIII, 2016, A\&A, 594, 13

Poole G. B., Fardal M. A., Babul A., McCarthy I. G., Quinn T., Wadsley J., 2006, MNRAS, 373, 881

Press W. H., Schechter P., 1974, ApJ, 187, 425

Ragone C. J., Merchán M., Muriel H., Zandivarez A., 2004, MNRAS, 350, 983

Randall S. W., Sarazin C. L., Ricker P. M., 2002, ApJ, 577, 579

Reiprich T. H., Basu K., Ettori S., Israel H., Lovisari L., Molendi S., Pointecouteau E., Roncarelli M., 2013, Space Sci. Rev., 177, 195

Ricker P., Sarazin C. L., 2001, ApJ, 561, 621

Rines K., Geller M. J., Diaferio A., Kurtz M. J., 2013, ApJ, 767, 15

Rizza E., Burns J. O., Ledlow M. J., Owen F. N., Voges W., Bliton M., 1998, MNRAS, 301, 328

Roediger E. et al., 2015a, ApJ, 806, 103

Roediger E. et al., 2015b, ApJ, 806, 104

Rozo E., Evrard A. E., Rykoff E. S., Bartlett J. G., 2014, MNRAS, 438, 62

Sanderson A. J. R., Ponman T. J., 2010, MNRAS, 402, 65

Schellenberger G., Reiprich T. H., 2015, A\&A, 583, L2

Schuecker P., Böhringer H., Collins C. A., Guzzo L., 2003, A\&A, 398, 867

Scoville N. et al., 2007, ApJS, 172, 1

Sheth R. K., Tormen G., 1999, MNRAS, 308, 119

Smith G. P., Kneib J.-P., Smail I., Mazzotta P., Ebeling H., Czoske O., 2005, MNRAS, 359, 417

Smith G. P. et al., 2010, A\&A, 518, L18

Smith G. P. et al., 2016, MNRAS, 456, L74

Spergel D. N. et al., 2003, ApJS, 148, 175

Springel V. et al., 2005, Nature, 435, 629

Taylor J. E., Babul A., 2005, MNRAS, 364, 515

Tinker J. L., Robertson B. E., Kravtsov A. V., Klypin A., Warren M. S., Yepes G., Gottlober S., 2010, ApJ, 724, 878

van den Bosch F. C., Tormen G., Giocoli C., 2005, MNRAS, 359, 1029

van den Bosch F. C., Jiang F., Hearin A., Campbell D., Watson D., Padmanabhan N., 2014, MNRAS, 445, 1713

Vikhlinin A., McNamara B. R., Forman W., Jones C., Quintana H., Hornstrup A., 1998, ApJ, 502, 558

Vikhlinin A. et al., 2009, ApJ, 692, 1060

Voit G. M., 2005, Rev. Mod. Phys., 77, 207

Weinmann S. M., van den Bosch F. C., Yang X., Mo H. J., 2006, MNRAS, 366,2

Woo J. et al., 2013, MNRAS, 428, 3306

Xu L., Rieke G. H., Egami E., Pereira M. J., Haines C. P., Smith G. P., 2015a, ApJS, 219, 18

Xu L., Rieke G. H., Egami E., Haines C. P., Pereira M. J., Smith G. P., 2015b, ApJ, 808, 159

Yang X., Mo H. J., van den Bosch F. C., Pasquali A., Li C., Barden M., 2007, ApJ, 671, 153

Zabludoff A. I., Mulchaey J. S., 1998, ApJ, 496, 39

Zabludoff A. I., Zaritsky D., Lin H., Tucker D., Hashimoto Y., Shectman S. A., Oemler A., Kirshner R. P., 1996, ApJ, 466, 104

Zhang Y.-Y., Finoguenov A., Böhringer H., Kneib J.-P., Smith G. P., Czoske O., Soucail G., 2007, A\&A, 467, 437

Zhang Y.-Y., Andernach H., Caretta C. A., Reiprich T. H., Böhringer H., Puchwein E., Sijacki D., Girardi M., 2011, A\&A, 526, 105

Zhao D. H., Jing Y. P., Mo H. J., Börner G., 2009, ApJ, 707, 354

Ziparo F. et al., 2014, MNRAS, 437, 458

APPENDIX A: A CATALOGUE OF OTHER X-RAY GROUPS 
Table A1. List of the other X-ray groups found within the $X M M$ images.

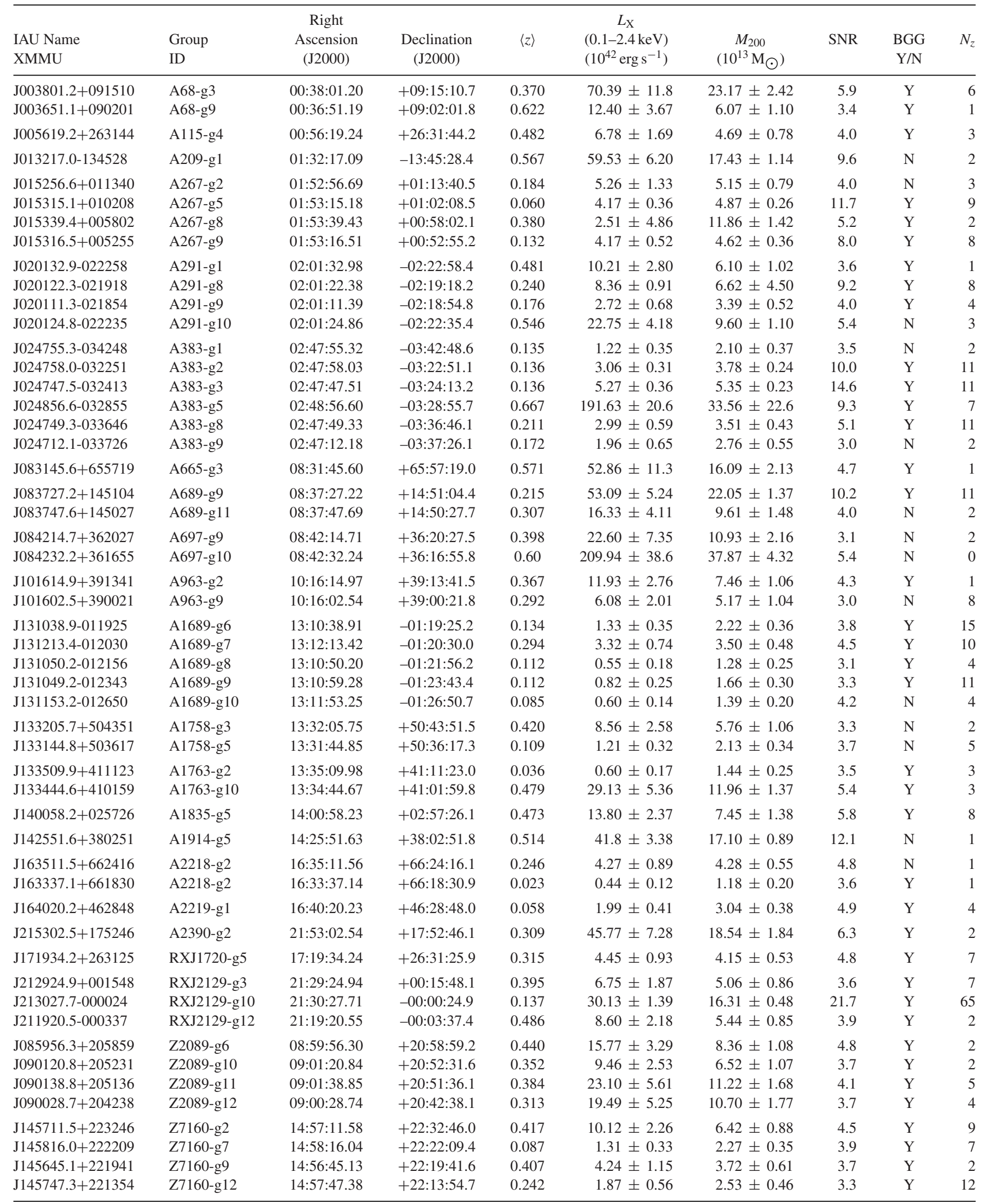

This paper has been typeset from a $\mathrm{T}_{\mathrm{E}} \mathrm{X} / \mathrm{LT}_{\mathrm{E}} \mathrm{X}$ file prepared by the author. 